\title{
NONLINEAR SECOND SOUND WAVES IN SUPERFLUID HELIUM: INSTABILITIES, TURBULENCE AND ROGUE WAVES
}

\author{
ANDREY N. GANSHIN \\ Department of Physics, Lancaster University, Lancaster LA1 4 YB, UK \\ Laboratory for Elementary-Particle Physics, Cornell University, NY 14853-5001, USA \\ ang43@cornell.edu \\ VICTOR B. EFIMOV \\ Institute of Solid State Physics, Chernogolovka, Moscow region, 142432, Russia \\ Department of Physics, Lancaster University, Lancaster, LA1 4YB, UK \\ efimov@issp.ac.ru \\ GERMAN V. KOLMAKOV \\ Department of Physics, New York City College of Technology, \\ CUNY, New York, NY 11201, USA \\ gvkolmakov@gmail.com \\ LEONID P. MEZHOV-DEGLIN \\ Institute of Solid State Physics, Chernogolovka, Moscow region, 142432, Russia \\ mezhov@issp.ac.ru \\ PETER V.E. McCLINTOCK \\ Department of Physics, Lancaster University, Lancaster, LA1 $4 Y B$, UK \\ p.v.e.mcclintock@lancaster.ac.uk
}

Received (to be inserted by publisher)

\begin{abstract}
Recent work on nonlinear second sound wave propagation and acoustic turbulence in superfluid ${ }^{4} \mathrm{He}$ is reviewed. Observations of direct and inverse turbulent energy cascades are described. The direct cascade arises due to the strong nonlinear dependence of the second sound wave velocity on its amplitude. The flux of energy injected at the driving frequency is transformed via successively higher harmonics until it is eventually attenuated by viscous dissipation at the short-wavelength edge of the spectrum. The onset of the inverse cascade occurs above a critical driving energy density, and it is accompanied by giant waves that constitute an acoustic analogue of the rogue waves that occasionally appear on the surface of the ocean. The theory of the phenomena is outlined and shown to be in good agreement with the experiments.
\end{abstract}

Keywords: Bifurcation, nonlinear waves, wave turbulence, second sound, superfluid helium

\section{Introduction}

Turbulence implies a highly excited state of a system with numerous degrees of freedom, usually character- 
ized by a directional energy flux through frequency scales [Kolmogorov, 1941; Zakharov et al., 1992]. The phenomenon arises in a number of variants and in a range of different contexts but, typically, energy is continuously fed into the system on one length scale (or frequency), cascades through the frequency scales without significant dissipation, and is finally dissipated on a smaller length scale (or higher frequency). It is an inherently nonequilibrium phenomenon in the sense that, if the driving force is removed, the turbulence dies away and disappears. Different kinds of turbulent behavior may be distinguished by the physical processes providing the energy pumping and energy transformation. In this sense we can usefully distinguish between hydrodynamic, convectional, and wave turbulence. They all are described by nonlinear equations, but their driving and inertial forces are different in nature. Although there are differing reasons for the instabilities in the motion, and differing mechanisms of energy transformation, the dissipative process is always the viscosity of the medium.

Hydrodynamic turbulence appears in a moving fluid when the inertial effects of its motion dominate substantially over viscous forces, i.e. when the Reynolds number Re is large. In the familiar scenario of 2D fluid flow around an obstacle (e.g. in a river), hydrodynamic turbulence appears as individual vortices, and evolves via a Karman vortex street towards fully developed turbulence [Frisch, 1995; Davidson, 2004]. Convective turbulence appears for steady heating of a liquid in a gravitational field, when the ratio between the Archimedean and viscous forces becomes large, i.e. when the Rayleigh number Ra is large. With increasing Ra there is a transformation from a thermal conductivity regime towards fully developed turbulence via regular flux structures like Rayleigh-Bénard cells and axisymmetric vortex flow [Incropera \& DeWitt, 1981].

Quantum turbulence (QT) in He II is an example of hydrodynamic turbulence, but with the important difference that the circulation around the vortex cores is singly-quantized, so that all elements of vortex are identical. Our current understanding [Vinen \& Niemela, 2002; Vinen, 2006] of QT in He II postulates a two-stage decay of turbulent energy initially in the form of quantized vortex lines of relatively large characteristic length. First there is a Richardson cascade towards vortex lines of smaller length scale, leading to the generation of Kelvin waves on the lines. Secondly, there is an analogous turbulent decay of the Kelvin waves until their wavelength is short enough that direct phonon generation becomes probable, finally dissipating the turbulent energy in the form of heat. This Kelvin wave decay is an example of wave turbulence (WT).

Our present paper treats another example of pure WT in He II, in the absence of vortices. We consider second sound WT, a form of acoustic turbulence that offers particular advantages in terms of experimental characterization and control, as we shall see below. Note that acoustic WT is also known to arise in e.g. phonon turbulence in solids [Tsoi, 2003], turbulence of sound waves in oceanic waveguides [Gurbatov et al., 2005], magnetic turbulence in interstellar gases [Bisnovatyi-Kogan \& Silich, 1995], and shock waves in the solar wind and their coupling with Earth's magnetosphere [Ryutova \& Tarbell, 2003]. In all cases there is a cascade-like transfer of turbulent energy towards the high frequency domain [Zakharov et al., 1992; Kolmakov et al., 2006], which is where the viscous dissipation mainly occurs.

In what follows, we review recent experimental investigations of acoustic WT created in a system of second sound standing waves in a high-quality resonator filled with He II. Second sound (temperature/entropy) waves are a macroscopic quantum effect seen in superfluids and highly anharmonic crystals [Landau \& Lifshitz, 1987; Khalatnikov, 1965; Ackerman et al., 1966; Putterman, 1974]. The properties of second sound in HeII have been extensively investigated in earlier studies, both experimentally and theoretically. More recently, attention has been focused on the nonlinear acoustic properties of He II [Iznankin \& Mezhov-Deglin, 1983; Borisenko et al., 1988; Mezhov-Deglin et al., 1980; Goldner et al., 1991; Nemirovskii, 1990]. These lead [Landau \& Lifshitz, 1987; Khalatnikov, 1965; Nemirovskii, 1990; Dessler \& Fairbank, 1956] to the formation of a planar or spherical shock wave [Temperley, 1951; Osborne, 1951; Atkin \& Fox, 1985] (temperature discontinuity) during the propagation of a finite-amplitude heat pulse in He II, at short distances from the source (heater). The velocity $u_{2}$ of a traveling second sound wave depends on its amplitude and, to a first approximation, can be written as

$$
u_{2}=u_{20}(1+\alpha \delta T),
$$

where $\delta T$ is the wave amplitude, $u_{20}$ is the velocity of a wave of infinitely small amplitude, and $\alpha$ is the 
nonlinearity coefficient of second sound, which is determined by the relation [Khalatnikov, 1965]

$$
\alpha=\frac{\partial}{\partial T} \ln \left(u_{20}^{3} \frac{C}{T}\right),
$$

$C$ is the heat capacity per unit mass of liquid helium at constant pressure, and $T$ is the temperature.

The nonlinearity coefficient $\alpha$ may be either positive or negative, depending on the temperature and pressure [Khalatnikov, 1965; Dessler \& Fairbank, 1956; Efimov et al., 2000]. Under the saturated vapor pressure (SVP), in the region of roton second sound (i.e. at $T>0.9 \mathrm{~K}$ ) the nonlinearity coefficient is positive $(\alpha>0)$ at temperatures $T<T_{\alpha}=1.88 \mathrm{~K}$ (like the nonlinearity coefficient of conventional sound waves in ordinary media), but it is negative $(\alpha<0)$ in the range $T_{\alpha}<T<T_{\lambda}$. Here $T_{\lambda}=2.176 \mathrm{~K}$ is the temperature of the superfluid-to-normal (He-II to He-I) transition. At $T=T_{\alpha}$ the nonlinearity coefficient passes through zero.

Provided $T \neq T_{\alpha}$, the nonlinear evolution leads to the creation of a shock in the profile of a travelling second sound pulse. During the propagation of a plane, one-dimensional wave of heating (compression) of second sound $(\delta T>0)$, the shock appears at the front of the propagating wave for temperatures $1 \mathrm{~K}$ $<T<T_{\alpha}$, and on the trailing edge of the wave for temperatures $T_{\alpha}<T<T_{\lambda}$ [Efimov et al., 2009]. The ease with which $\alpha$ can be adjusted (by alteration of $T$ ) and the large values it can take make second sound in HeII ideal as a test-bed for the study of nonlinear wave interactions and associated phenomena, including WT. The frequency of second sound depends on its wave vector $k$ as [Tyson \& Douglass, Jr., $1968]$

$$
\omega=u_{20} k\left[1+\lambda_{0} \xi^{2}(T) k^{2}+\ldots\right]
$$

where $\xi(T)=\xi_{0}\left(1-T / T_{\lambda}\right)^{-2 / 3}, \xi_{0} \sim 2-3 \mathrm{~A}$, and $\lambda_{0} \sim 1$. We emphasize, however, that the dispersion of second sound is only significant within a close vicinity of the superfluid transition (i.e. at $T_{\lambda}-T<1 \mu \mathrm{K}$ ) and is extremely weak in the temperature range $T<2.1 \mathrm{~K}$ relevant to the present investigations.

Our most recent studies demonstrate that wave energy can flow, not only towards higher frequencies as in the conventional scenario [Kolmakov et al., 2006], but also in the opposite direction [Ganshin et al., 2008a], leading to the appearance of waves at lower frequencies. This leads to formation of the acoustic analogue of the "rogue" (giant) waves that sometimes appear on the ocean and which can endanger even large ships. Realistic experiments on oceanic rogue waves are of course almost impossible, because they probably require hundreds of miles of open sea to build up and decay. As we will see, however, second sound provides an excellent modeling medium for studies of the nonlinear wave interactions believed to be involved in the generating mechanism [Dyachenko \& Zakharov, 2005], enabling the fundamental ideas to be tested in the laboratory under controlled conditions.

We have found that this flux of energy towards lower frequencies (inverse cascade) can coexist with the direct cascade. The backflow appears to be attributable to a decay instability, i.e. nonlinear decay of the wave into two waves of lower frequency governed by the conservation law [Zakharov et al., 1992]

$$
\omega_{1}=\omega_{2}+\omega_{3} .
$$

where $\omega_{i}=u_{20} k_{i}$ is the frequency of a linear wave of wave vector $k_{i}$. The instability results in the creation of subharmonics and can produce a substantial increase of low-frequency wave amplitude. A similar parametric process, but caused by 4-wave processes (modulation instability), is thought [Dyachenko \& Zakharov, 2005] to be responsible for the conversion of the energy in ordinary wind-driven waves on the ocean to take the form of rogue waves; this mechanism differs from an alternative explanation proposed more recently [Solli et al., 2007] involving the scattering of nonlinear waves on a continuous noisy background. Comparable decay instabilities have been studied for e.g. spin waves [Anderson \& Suhl, 1955; L'vov, 1994; Matsushita et al., 1996], magnetohydrodynamic waves in plasma [Spangler et al., 1997], and interacting first and second sound waves in superfluid helium near the superfluid transition [Rinberg et al., 1996].

Through application of a Hamiltonian formalism to the nonlinear wave system (see Sec. 4), we demonstrate that formation of the high- and low-frequency harmonics in the turbulent spectrum is caused by instabilities of the nonlinear waves against the confluence process leading to increased resultant wave frequency, and against wave decay where the resultant wave frequency decreases. We also show that confluence 
has an effectively zero threshold amplitude and thus can always occur (though the resultant amplitudes of high-frequency harmonics may sometimes be small due to the non-zero viscous damping). In contrast, the decay process has a finite threshold amplitude and its onset has the character of a bifurcation.

In Sec. 2 we outline the experimental techniques used for the investigations. In Sec. 3 we describe results on the forward and inverse turbulent energy cascades, and we discuss their implications is Sec. 4. Finally, in Sec. 5, we summarise our conclusions. We emphasise that the research programme is still in progress and that additional significant results are to be anticipated, especially in relation to transient effects and the kinetics of these turbulent phenomena which we mention only briefly, for completeness, in the present review.

\section{Experimental Techniques}

The experimental arrangements [Efimov et al., 2006] are shown schematically in Fig. 1. The cryoacoustical resonator was formed from a cylindrical quartz tube of nominal length $L=7 \mathrm{~cm}$ and internal diameter $D=$ $1.5 \mathrm{~cm}$, filled with superfluid helium. The low-thermal-inertial film heater and bolometer were deposited on the surfaces of flat glass plates capping the ends of the tube. The heater could be driven from a voltage pulse generator for experiments with solitary traveling waves, or by a harmonic voltage generator for experiments with quasi-one-dimensional standing waves.

For the experiments to be described below, the heater of resistance $R$ was driven by a sinusoidal voltage $U=U_{0} \sin \omega t$. The corresponding total heat flux is

$$
W_{t o t}=\frac{U^{2}}{R}=\left(\frac{U_{0}^{2}}{R}\right) \times \sin ^{2}(\omega t)=\left(\frac{U_{0}^{2}}{2 R}\right) \times(1-\cos (2 \omega t))=W_{0}+W .
$$

There are both constant, $W_{0}$, and periodic, $W$, components. The frequency of the harmonic voltage generator was set within the range $0.1-100 \mathrm{kHz}$ so that the second sound frequency (at twice the generator frequency) was close to that of a longitudinal resonance in the cell. The amplitude $\delta T$ of the resultant standing wave could be varied from $0.05 \mathrm{mK}$ up to a few $\mathrm{mK}$ by adjustment of the power to the heater.

The Mach number in the second sound standing wave can reach $\mathrm{M}=\left|v_{n}-v_{s}\right| / u_{20} \sim 10^{-4}-10^{-2}$, where $v_{n}$ and $v_{s}$ are the normal and superfluid velocities. The proper Reynolds number, defined [Davidowitz et al., 1995] as

$$
\operatorname{Re}=\frac{\alpha u_{20}}{\gamma_{\omega}}\left(\frac{\partial \delta T}{\partial x}\right) \sim \alpha Q \delta T,
$$

can be changed from 1 to 90 (here $\gamma_{\omega}$ is the damping coefficient of a second sound wave of frequency $\omega$, evaluated from the $Q$-factor of the resonator). It allows us to study the transitions from the linear regime to the nonlinear one, and from there to turbulence, in the system of second sound waves as the driving amplitude is being increased.

The measured density $W$ of the heat flux into the liquid is subject to systematic uncertainties of up to $\pm 10 \%$ associated with estimation of the resonator cross-section and the resistances of the leads, and possible small inhomogeneities in the heater film thickness. The $Q$-factor of the resonator was determined from the widths of longitudinal resonances at small heat fluxes $W \leq 1 \mathrm{~mW} / \mathrm{cm}^{2}$ (nearly linear regime). The result was $Q \sim 3000$ for resonance numbers $20<p<100$, decreasing to about 500 at frequencies below $500 \mathrm{~Hz}(p<5)$.

Use of a high- $Q$ resonator in this way is advantageous because it enables us to create nonlinear second sound standing waves of high amplitude $(\delta T \sim 1 \mathrm{mK})$, while the heat input at the source remains relatively small $W<50 \mathrm{~mW} / \mathrm{cm}^{2}$. Hence we avoid possible complications [Goldner et al., 1991; Davidowitz et al., 1995] due to vortex creation in the bulk He II or nonlinear phenomena at the heater/superfluid interface. The second sound signal detected by the bolometer was Fourier-analyzed and its power spectrum was computed.

\section{Acoustic Turbulence of Second Sound Waves in He II}

We now consider some recent measurements [Kolmakov et al., 2006; Ganshin et al., 2008a; McCintock et al., 2008; Efimov et al., 2008a] of second sound standing waves that that have demonstrated the occurrence 


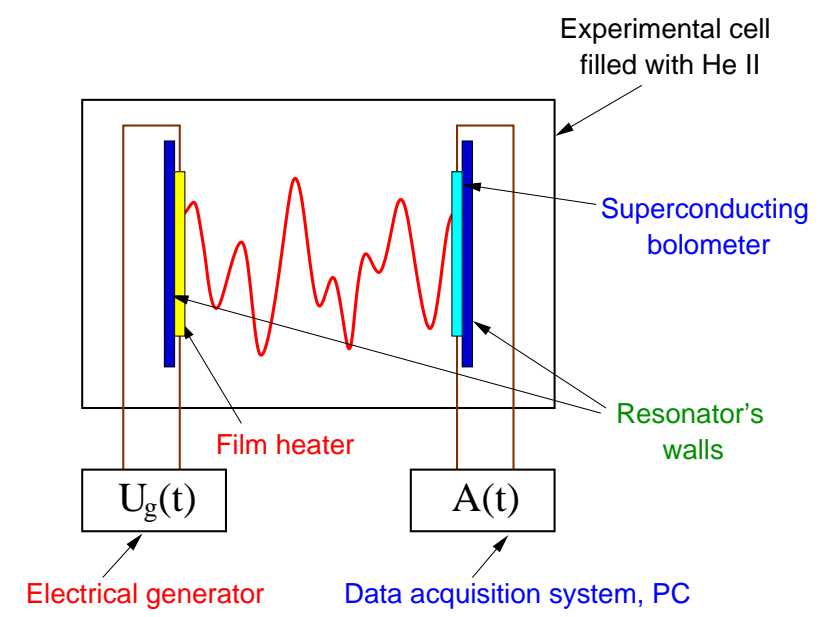

Fig. 1. Schematic diagram illustrating the experimental arrangements. The cylindrical walls of the resonator are not shown.
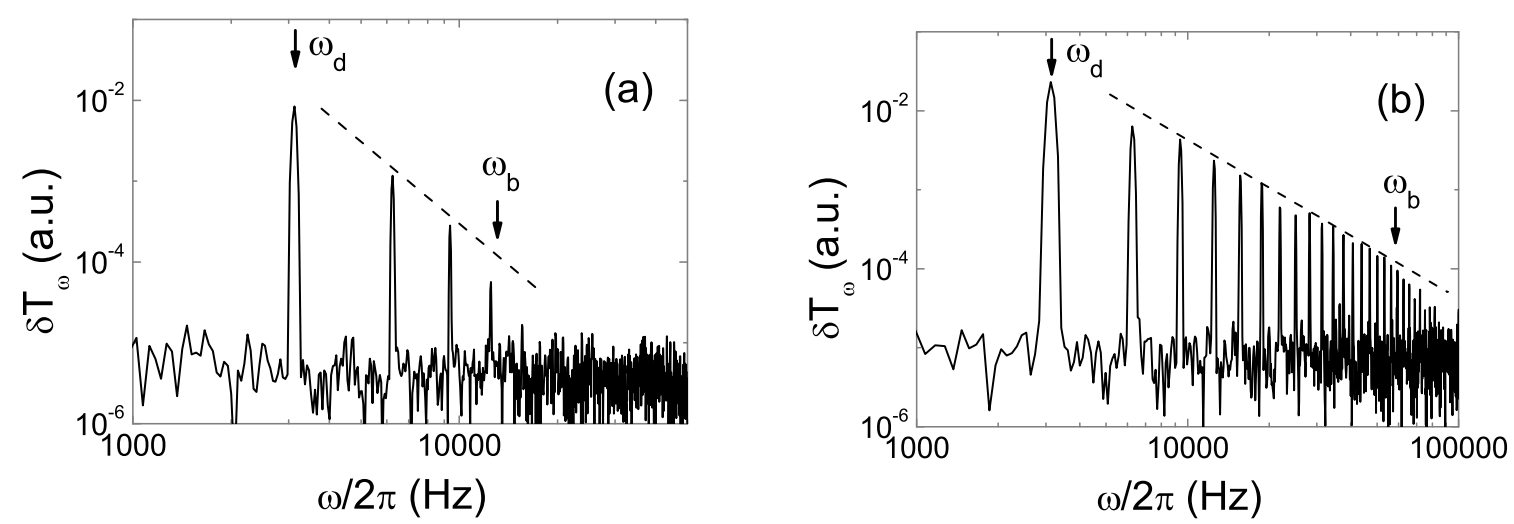

Fig. 2. Change in the power spectrum of second sound standing waves as the AC heat flux density is increased from (a) $W=4 \mathrm{~mW} / \mathrm{cm}^{2}$ to (b) $25 \mathrm{~mW} / \mathrm{cm}^{2}$. The system was driven on its $31 \mathrm{st}$ resonance, at a temperature close to $2.08 \mathrm{~K}$. The dashed line in (a) is a guide to the eye, whereas that in (b) corresponds to $\delta T \propto \omega^{-1.7}$. The arrows indicate the positions of the fundamental spectral peak formed at the driving frequency $\omega_{d}$ and of the high-frequency edge $\omega_{b}$ of the inertial frequency range. From Kolmakov et al. [2006]

of both the forward and inverse energy cascades in acoustic WT. Again, we restrict the discussion to the range of heat fluxes $W \leq 50 \mathrm{~mW} \mathrm{~cm}^{-2}$ for which we can neglect possible formation of quantized vortices.

\subsection{Observation of a direct Kolmogorov-like cascade}

Fig. 2 shows the evolution of the second sound wave spectrum with increasing AC heat flux density $W$ from the heater, measured near $2.08 \mathrm{~K}$ while driving at the frequency of the 31st resonance [Kolmakov et al., 2006]. For small $W \leq 4 \mathrm{~mW} / \mathrm{cm}^{2}$ we observed a nearly linear regime of wave generation, where a small number of harmonics of the driving frequency were excited due to nonlinearity (see Fig. 2(a)), and the shape of the recorded signal remained nearly sinusoidal. An increase of the excitation level above $10 \mathrm{~mW} / \mathrm{cm}^{2}$ led to visible deformation of the signal shape and to the generation of a large number of high-frequency harmonics in the second sound wave spectrum as shown in Fig. 2(b). The dependence of wave amplitude $\delta T$ on the excitation level $W$ deviates from a linear relationship above $\delta T \sim W_{d}^{2}$ (see Fig. 1 of Efimov et al. [1998]

It is evident from Fig. 2 that the main spectral peak (marked by the left arrow) appears at the driving frequency $\omega_{d}$, and that higher-frequency peaks appear at its harmonics $\omega_{n}=n \times \omega_{d}$ with $n=2,3, \ldots$. Fig. 2(b) shows that a cascade of waves is formed over frequencies ranging up to $80 \mathrm{kHz}$, i.e. up to a 

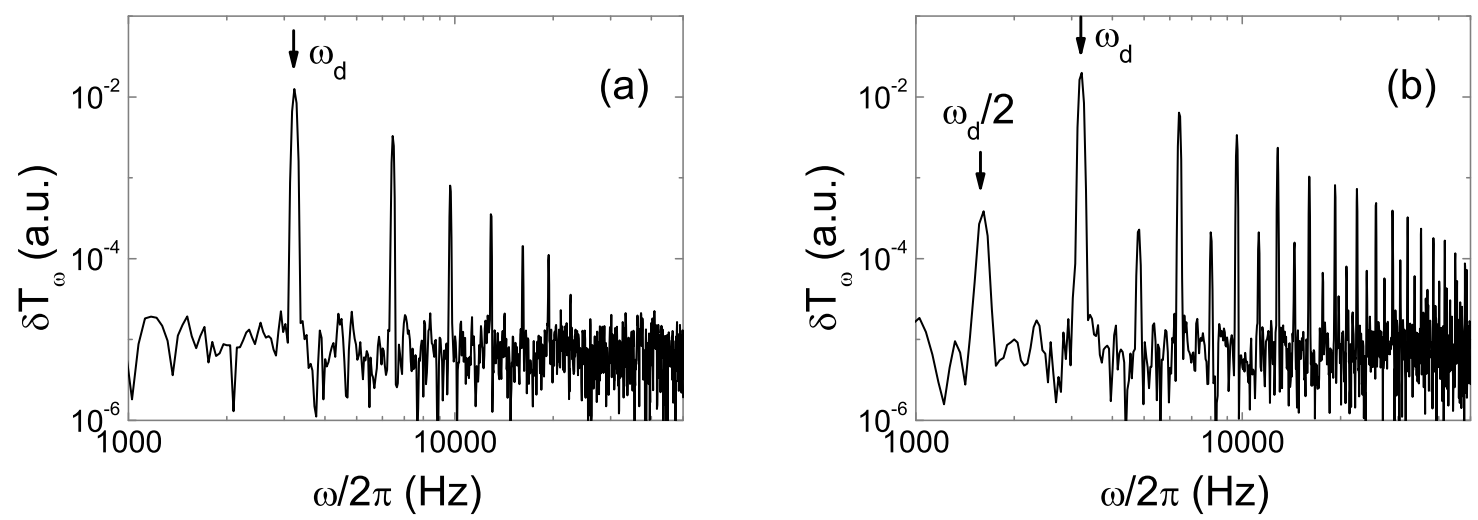

Fig. 3. Second sound wave spectra measured when driving on the frequency of the $32 \mathrm{nd}$ resonance with (a) $W=4 \mathrm{~mW} / \mathrm{cm}^{2}$ and (b) $W=16 \mathrm{~mW} / \mathrm{cm}^{2}$. The arrows indicate the fundamental peaks at the driving frequency $\omega=\omega_{d}$ and a subharmonic formed at $\omega=\omega_{d} / 2$. The temperature was close to $2.08 \mathrm{~K}$. From Ganshin et al. [2008b]

frequency $25 \times$ the driving frequency. Fig. 2(b) also shows that the dependence of peak height on frequency is power-law-like in form, $\delta T_{\omega}=$ const $\times \omega^{-s}$ for frequencies lower than some cut-off frequency $\omega_{b}$ that rises with increasing $W$. Note there are systematic uncertainties of around $\pm 10 \%$ in $s$ and $\omega_{b}$ as extracted from plots of this kind, depending on the range of $\omega$ through which the straight line is drawn. The scaling index tends towards $s \approx 1.5$ for sufficiently high AC heat flux densities $W>12 \mathrm{~mW} / \mathrm{cm}^{2}$ (i.e. for the developed cascade).

These spectra are evidently attributable to the cascade transfer of wave energy through the frequency scales on account of nonlinearity, thus establishing an energy flux in $K$-space starting from the driving frequency and directed towards the high-frequency domain. In accordance with the basic ideas presented in Refs. [Kolmogorov, 1941; Zakharov et al., 1992; L'vov, 1994] we may infer that, for relatively high amplitude driving, we are observing acoustic turbulence in the system of second sound waves within its inertial (nondissipative) range of frequencies. This direct cascade is similar to the Kolmogorov distribution of fluid velocity over frequency in the bulk of a classical fluid [Kolmogorov, 1941].

We also observed that, when the AC heat flux density was raised above a critical value at even resonance numbers $p>30$, a spectral peak appeared at the frequency equal to half the driving frequency (i.e. formed on the left of the fundamental peak) and at its harmonics. Fig. 3 shows the evolution of the wave spectrum with increasing AC heat flux density when driving on the 32nd resonance [Ganshin et al., 2008b]. It can be seen that, for a relatively small heat flux density of $W=4 \mathrm{~mW} / \mathrm{cm}^{2}$, the wave spectrum shown in Fig. 3(a) is quite similar to that observed under similar conditions when driving on the 31st resonance (i.e. at the nearest odd numbered resonance), cf. Fig. 2(a). Formation of the low-frequency harmonic (subharmonic) at $\omega=\omega_{d} / 2$ at $W=16 \mathrm{~mW} / \mathrm{cm}^{2}$ can clearly be seen in Fig. 3(b). It was found experimentally that for $30<p<96$ the threshold for subharmonic generation was about $10 \mathrm{~mW} / \mathrm{cm}^{2}$ (see Fig. 6 below).

The generation of subharmonics may be attributed to a decay instability of the periodic wave, see Sec. 4.2. In accordance with general theory [Kolmogorov, 1941; Zakharov et al., 1992; L'vov, 1994], the instability is controlled mainly by nonlinear decay of the wave into two waves of lower frequency, and by the opposite process i.e. by the confluence of two waves to form one wave. The energy (or frequency) conservation law for this 3-wave process is given by Eq. (3). The case shown in Fig. 3(b) evidently corresponds to generation of subharmonics with $\omega_{2}=\omega_{3}=\omega_{d} / 2$.

As shown in Fig. 2(a), the high-frequency cutoff in the wave spectrum due to viscosity manifests itself as an abrupt decrease in the amplitudes of the harmonics at $W<10 \mathrm{~mW} / \mathrm{cm}^{2}$, and as a change in gradient of the spectrum at higher $W$ (Fig. 2(b)) when plotted on double-log scales, which occurs at some characteristic frequency $\omega_{b}$. At $\omega \sim \omega_{b}$ the almost nondissipative transfer of wave energy changes to viscous damping of the waves (cf. observations of the high-frequency edge of the inertial range of frequencies of capillary turbulence on the surface of liquid hydrogen [Kolmakov et al., 2009]). It results in the observed 


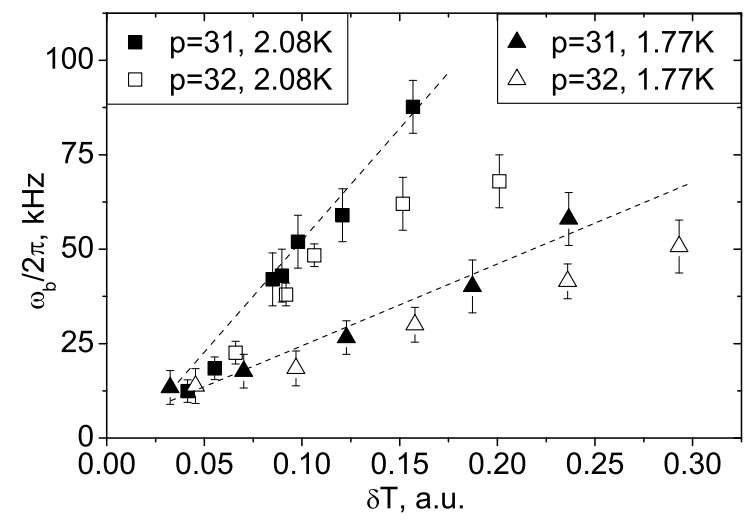

Fig. 4. Dependence of the viscous cutoff frequency $\omega_{b} / 2 \pi$ on the amplitude $\delta T$ of the standing wave for different temperatures $T$ and resonance numbers $p$. Dashed lines indicate the results of our numerical computations; data points represent experimental measurements. From Kolmakov et al. [2006]

faster reduction of sound amplitude at frequencies $\omega>\omega_{b}$.

Fig. 4 shows the measured dependence [Kolmakov et al., 2006] of the boundary frequency $\omega_{b} / 2 \pi$ on the standing wave amplitude $\delta T$. It can be seen that the inertial range extends further towards higher frequencies as the driving force increases. When driving at resonant frequencies with odd resonance numbers $p$, with sufficiently large driving amplitudes, the boundary frequency depends nearly linearly on wave amplitude

$$
\omega_{b} / 2 \pi \approx \operatorname{const}\left(T, \omega_{d}\right) \times \delta T,
$$

(the filled symbols in Fig. 4). Our numerical calculations [Kolmakov et al., 2006] are in agreement with this result.

When driving at even $p$ (shown by the open symbols in Fig. 4) the boundary frequency is significantly lower than that measured when driving at the nearest odd resonance number, with $W>10 \mathrm{~mW} / \mathrm{cm}^{2}$. It seems natural to infer that his reduction is connected to a change in the mechanism of energy relaxation in the wave system caused by the generation of subharmonics with frequencies below $\omega_{d}$.

Fig. 4 shows that the energy balance in the wave system is highly nonlocal in $K$ space: energy is pumped into the system in the low-frequency (long-wave) domain and it flows to the high frequency (short-wave) domain where it is absorbed by dissipative mechanisms.

\subsection{Inverse Energy Cascade of Acoustic Turbulence}

When the driv was applied at sufficiently high AC heat flux densities $W>10 \mathrm{~mW} / \mathrm{cm}^{2}$ and at resonance numbers $p>50$, multiple subharmonics were generated in the low-frequency spectral domain $\omega<\omega_{d}$. Fig. 5 presents typical results obtained when driving at a relatively high resonant frequency $\omega_{d}$ (the 96 th longitudinal resonance of the cell). Those in Figs. 5(a),(b) reproduce our earlier observation of the direct Kolmogorov-like cascade [Kolmakov et al., 2006], when driving close to resonance. Figs. 5(c)-(f) show the evolution of the wave shape and spectrum with small changes in the detuning of the drive frequency of up to $\Delta=\left(\omega_{d}-\omega_{p}\right) / \omega_{p}=5 \times 10^{-4}$. Of particular interest for what follows, tiny shifts in driving frequency result in marked changes in the shape and power spectrum of the standing wave. The formation of spectral peaks near $\frac{1}{2}, \frac{1}{3}$ and $\frac{2}{3} \times \omega_{d}$ satisfy (3) with $\omega_{1}=\omega_{d}$, supporting our inference that the instability is controlled mainly by 3 -wave interactions. We comment that the subharmonic generation giving rise to the inverse cascade bears striking similarities to the Feigenbaum period-doubling route to chaos [Devaney, 2000]. Huge distortions of an initially periodic signal occur as the driving frequency changes, as shown in Fig. 5(c),(e): when the instability develops, the signal remains nearly periodic, but its characteristic period becomes considerably longer than the fundamental period of the driving force (shown by horizontal arrows 

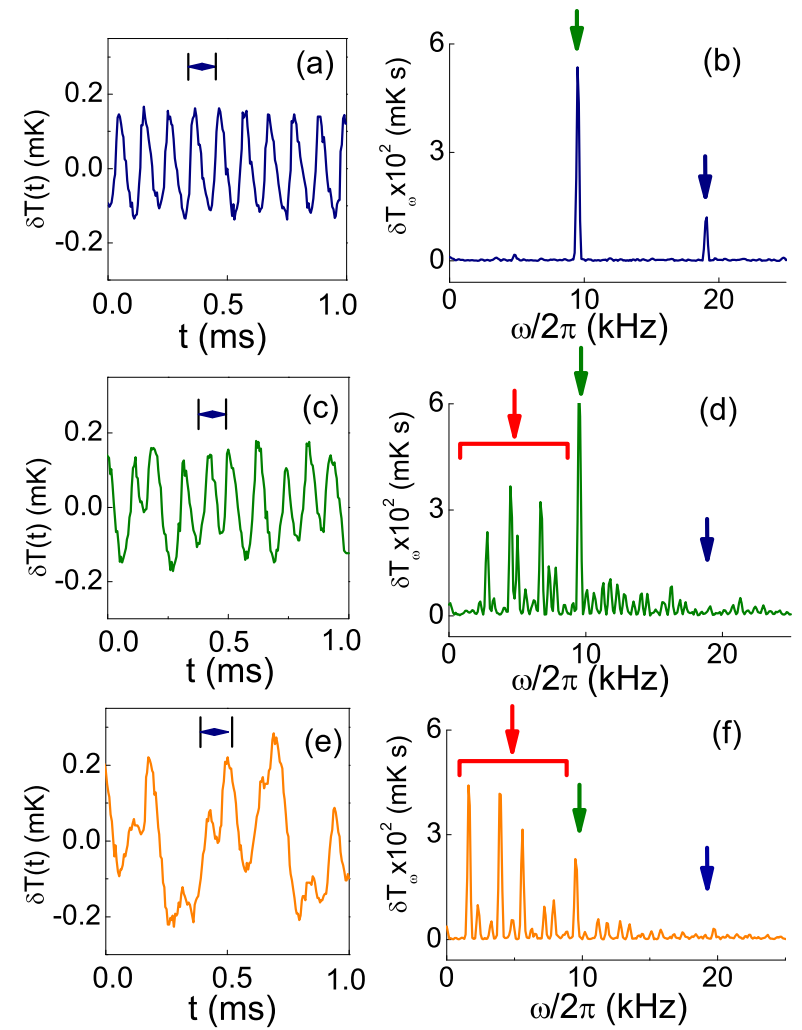

Fig. 5. Evolution of the observed second sound standing wave with increasing (small) positive detuning of the drive frequency $\omega_{d}$ near the 96th resonance. The left-hand column shows the steady state wave shape, and the right-hand column shows the corresponding power spectra for: (a),(b) $\omega_{d} / 2 \pi=9530.8 \mathrm{~Hz}$; (c),(d) $9532.4 \mathrm{~Hz}$; and (e),(f) $9535.2 \mathrm{~Hz}$. The AC heat flux density was $W=42 \mathrm{~mW} / \mathrm{cm}^{2}$. The temperature $T=2.08 \mathrm{~K}$ corresponded to negative nonlinearity. The fundamental and first harmonic in (b),(d),(f) are indicated by vertical (green and blue) arrows; the low-frequency domain where the subharmonics appear is indicated by the vertical (red) arrows and horizontal ranges in (d),(f). The horizontal arrows in (a),(c),(e) indicate the fundamental period of the wave at the driving frequency. From Efimov et al. [2008a]

in the left-hand column of Fig. 5). The amplitude of the corresponding lower-frequency waves can reach more than twice that at the driving frequency.

We can use the relative energy content of the low-frequency part of the spectrum $\omega<\omega_{d}$,

$$
E_{L F}=\frac{1}{2}\left(\frac{\partial C}{\partial T}\right) \sum_{\omega<\omega_{d}}\left|\delta T_{\omega}\right|^{2}
$$

as an indicator for characterising the instability quantitatively. For small $W$ we did not observe any subharmonic generation at all [Kolmakov et al., 2006]; then, above a critical flux $W_{c}, E_{L F}$ rose rapidly [Ganshin et al., 2008a], as shown in Fig. 6, suggesting that the phenomenon is of a threshold character. At $T=T_{\alpha}=1.88 \mathrm{~K}$ for which $\alpha$ vanishes [Dessler \& Fairbank, 1956], no subharmonics were observed, regardless of the magnitude of $W$, thus confirming the crucial importance of nonlinearity. For $W$ above 10.4 $\mathrm{mW} / \mathrm{cm}^{2}$, we observed a distortion of the signal similar to that shown in Fig. 5(c) and the formation of a few subharmonics. Further increase of $W$ above $20 \mathrm{~mW} / \mathrm{cm}^{2}$ led to the generation of multiple subharmonics. These phenomena appear under conditions where the energy cascade towards the high frequency domain (i.e. direct cascade, with a Kolmogorov-like spectrum [Kolmakov et al., 2006; Falkovich \& Meyer, 1996]), is already well-developed. 

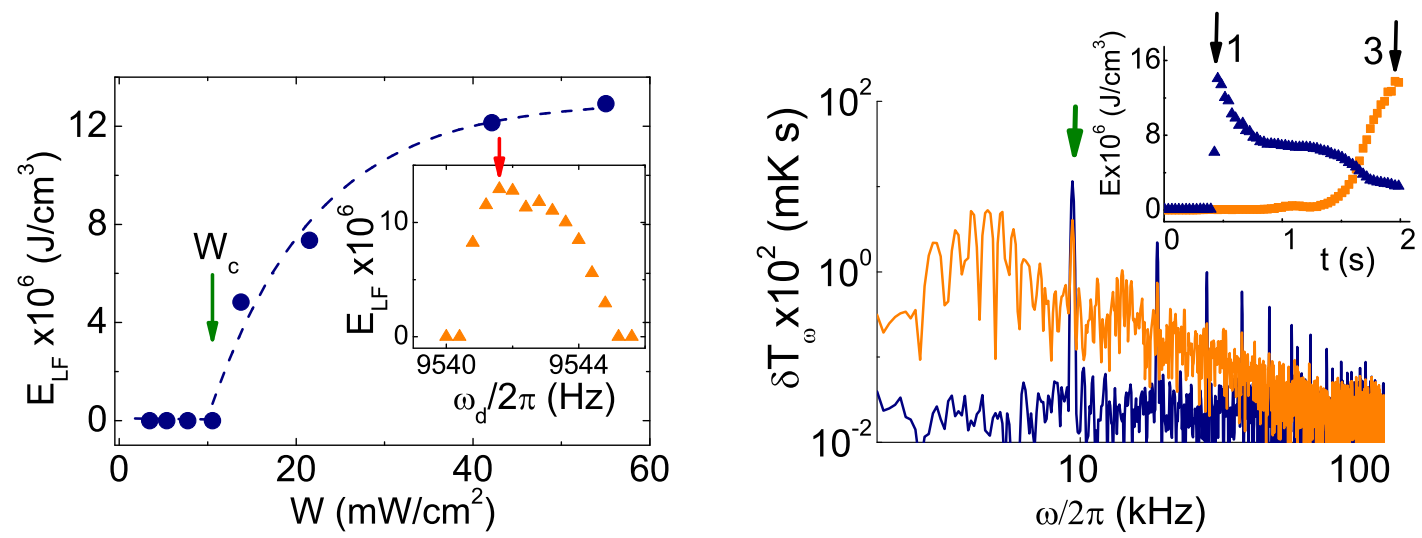

Fig. 6. Left: The energy $E_{L F}$ contained in the low-frequency part of the steady-state spectrum as a function of the AC heat flux density $W$, while driving near to the 96 th resonance for $T \simeq 2.08 \mathrm{~K}$. The threshold value of $W$, marked by the (green) arrow, was $W_{c}=10.4 \mathrm{~mW} / \mathrm{cm}^{2}$. The points are from experiment; the dashed line is a guide to the eye. Inset: the dependence of $E_{L F}$ on $\omega_{d}$, measured for $W=55.6 \mathrm{~mW} / \mathrm{cm}^{2}$; the (red) arrow labels the maximum value of $E_{L F}$, which is taken to the main figure. Right: Instantaneous spectra in frames 1 and 3 of Fig. 7. The lower (blue) spectrum, for frame 1, shows the direct cascade only; the upper (orange) spectrum, for frame 3, shows both the direct and inverse cascades. The (green) arrow indicates the the fundamental peak at the driving frequency. Inset: evolution of the wave energy in the low-frequency and high frequency domains is shown by the (orange) squares and (blue) triangles respectively; (black) arrows mark the positions of frames 1 and 3. From Ganshin et al. [2008a]

\subsection{Transient processes}

The above results all correspond to steady-state regimes of the wave system. In Figs. 6 (bottom) and 7 we illustrate the transient processes observed after a step-like shift in the driving frequency, from an initial frequency far from any resonance to the $96 \mathrm{th}$ resonance frequency, for $W=42.1 \mathrm{~mW} / \mathrm{cm}^{2}, T=2.08 \mathrm{~K}$. We find that the harmonics of the drive in the are formed very quickly high-frequency spectral domain, but that the formation of subharmonics is a much slower process: it took $\sim 0.5 \mathrm{~s}$ here, and can reach several tens of seconds under some conditions [Ganshin et al., 2008a]. The results shown in the inset of Fig. 7 reveal that, as the instability develops, isolated "rogue waves" appear in the signal. As time evolves, the rogue waves appear more frequently and, in the later stages, they merge resulting in the strong low-frequency modulation of the signal observed in the steady-state measurements (Fig. 5).

With the appearance of the subharmonics, there is a marked reduction in the energy content of the high-frequency spectral domain [Ganshin et al., 2008a]. The reason is that much of the energy injected at the driving frequency $\omega_{d}$ then flows towards the low frequency domain $\omega<\omega_{d}$ leading to an accumulation of wave energy there, with a corresponding increase in wave amplitude. The reduction of wave amplitude seen in the high frequency spectral domain clearly indicates the onset of a sharing of the flux between the direct and inverse energy cascades. The decrease in energy at high frequencies in the interval $0.397 \mathrm{~s}<t<1.3 \mathrm{~s}$ is attributable to relaxation processes in the direct cascade. The corresponding redistribution of wave energy between the direct and inverse cascades starts at about $t=1.3 \mathrm{~s}$. The transient evolution shown in Figs. 6 (bottom) and 7 is incomplete: the forward and inverse energy fluxes are still changing at $t=2 \mathrm{~s}$, implying further relaxation oscillations at longer times (inaccessible with our present equipment): the transient dynamics is highly complex, and a full characterisation will require further work. For there to be a flux of energy towards low frequencies, there must of course be some dissipative mechanism in the low frequency limit. Given that bulk second sound damping is negligibly small in this frequency range [Zinov'eva, 1957], we believe that the dissipation is probably attributable to viscous drag of the normal fluid component on the resonator walls: this would be consistent with the marked decrease of the resonator $Q$-factor seen below $3 \mathrm{kHz}$. Hysteresis occurs between increasing and decreasing frequency scans. The width of the hysteretic region, i.e. the region where the low frequency sound waves are metastable, was found to be less than the viscous width of the resonance. 


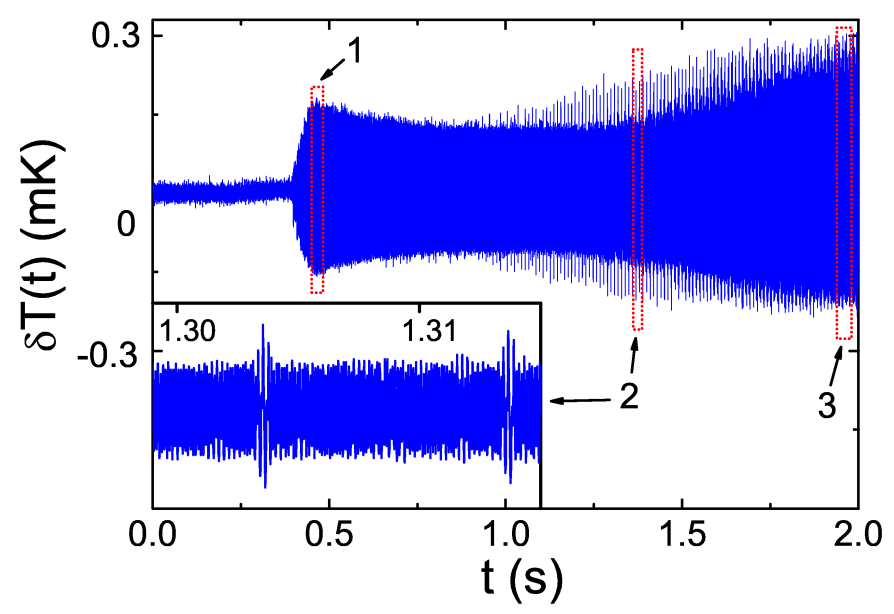

Fig. 7. Transient evolution of the 2nd sound wave amplitude $\delta T$ after a step-like shift of the driving frequency to the $96 \mathrm{th}$ resonance at time $t=0.397 \mathrm{~s}$. The $\mathrm{AC}$ heat flux density was $W=42.1 \mathrm{~mW} / \mathrm{cm}^{2}$, the temperature was $T=2.08 \mathrm{~K}$. The signals in frames 1 and 3 are similar to those obtained in the steady-state measurements, Fig. 5(a) and (e) respectively. The formation of isolated rogue waves is clearly evident. Inset: examples of rogue waves, enlarged from frame 2. From Ganshin et al. [2008a]

It has been established both theoretically [Pushkarev \& Zakharov, 1996; Kartashova, 2010] and experimentally [Abdurakhimov et al., 2010] that the discrete character of the frequency spectrum in a cell of finite size can sometimes affect strongly the wave distribution at high frequencies. In the case of the direct cascade in a quasi-one-dimensional acoustic resonator, the discreteness of the resonant frequency spectrum is not of essential importance: because of the linear dispersion relation of the sound waves, $\omega \propto k$, two resonant waves of frequencies $\omega_{1}$ and $\omega_{2}$ generate a wave of frequency $\omega_{3}=\omega_{1}+\omega_{2}$, which is also at resonance. For the inverse cascade, however, the discreteness of the resonance spectrum is important during the initial stages in the development of the decay instability. As we demonstrate in Sec. 3.1, the generation of low-frequency harmonics differs depending on whether the driving at an even or odd resonance number, cf. Figs. 2, 3. When driving far above the instability threshold, multiple low-frequency subharmonics are excited and the spectrum consequently becomes quasi-continuous, as shown in Fig.6.

\section{Theoretical Considerations}

\subsection{Hamiltonian formulation of superfluid hydrodynamics in superfluid helium}

\subsubsection{Superfluid hydrodynamics}

As mentioned above in Sec. 1, Hamiltonian formalism plays a central role in the subsequent consideration of kinetic processes in superfluid liquid. For the reader's convenience, we now sketch the Hamiltonian formulation of the superfluid hydrodynamics equations [Pokrovskii \& Khalatnikov, 1976; Khalatnikov et al., 1995; Kolmakov, 2003; Kolmakov et al., 2004, 2006, 2009]. Landau's well-known hydrodynamics equations for a quantum liquid with total density $\rho$ and densities $\rho_{s}$ and $\rho_{n}$ for its superfluid and normal components are [Khalatnikov, 1965]

$$
\begin{array}{ll}
\frac{\partial \rho}{\partial t}+\operatorname{div} \mathbf{j}=0, & \frac{\partial S}{\partial t}+\operatorname{div}\left(S \boldsymbol{v}_{n}\right)=0, \\
\frac{\partial \boldsymbol{v}_{s}}{\partial t}+\nabla\left(\mu+\frac{1}{2} \boldsymbol{v}_{s}^{2}\right)=0, & \frac{\partial j_{i}}{\partial t}+\frac{\partial \Pi_{i k}}{\partial x_{k}}=0 .
\end{array}
$$


Here $S$ is the entropy per unit volume, $\boldsymbol{v}_{s}$ and $\boldsymbol{v}_{n}$ are the velocities of superfluid and normal motion, $\boldsymbol{j}=\rho_{s} \boldsymbol{v}_{s}+\rho_{n} \boldsymbol{v}_{n}$ is the mass flux, $\Pi_{i k}=p \delta_{i k}+\rho_{s} v_{s i} v_{s k}+\rho_{n} v_{n i} v_{n k}$ is the tensor of the momentum flux, $\mu=\partial \varepsilon / \partial \rho$ is the chemical potential pre unit mass, and $\varepsilon$ is the energy per unit volume. Equations (6) are respectively the continuity equations for the density, entropy and momentum of the fluid, and the equation for the superfluid velocity. The damping terms in Eqs. (6) are omitted. In this review, we restrict our consideration to processes for which thermal expansion of the fluid is not an essential feature, and the terms proportional to the thermal expansion coefficient $\kappa_{T}=(T / \rho)(\partial \rho / \partial T)$ of the fluid may therefore be neglected. For superfluid ${ }^{4} \mathrm{He}$, this coefficient is in fact very small, with $\kappa_{T} \sim 10^{-2}$ for temperatures $T<2.15 \mathrm{~K}$.

In a non-stationary state, the density, entropy and velocity of the superfluid are not equal to their equilibrium values. For an infinitely small deviation from equilibrium, Eqs. (6) are reduced to two linear wave equations for perturbations of the density $\delta \rho$, and the entropy per unit mass $\delta \sigma$ [Khalatnikov, 1965],

$$
\frac{\partial^{2} \delta \rho}{\partial t^{2}}=u_{1}^{2} \Delta \delta \rho, \quad \frac{\partial^{2} \delta \sigma}{\partial t^{2}}=u_{2}^{2} \Delta \delta \sigma .
$$

Here $u_{1}=(\partial p / \partial \rho)^{1 / 2}$ and $u_{2}=\left(\rho_{s} \sigma^{2} T / \rho_{n} c\right)^{1 / 2}$ are the first and second sound velocities, $\sigma=S / \rho$, and $c$ is the heat capacity per unit mass. The linear equations (7) describe the propagation of two types of wave in the bulk superfluid: density waves in which $\delta \sigma=0$ at velocity $u_{1}$, and entropy waves in which $\delta \rho=0$ at velocity $u_{2}$. They are known as first sound and second sound, respectively [Khalatnikov, 1965]. In second sound, the fluid density remains constant but the temperature varies with coordinates and time as $\delta T=(\partial T / \partial \sigma) \delta \sigma$ [Lifshitz, 1944]. The possibility of propagating temperature waves that are only weakly dissipative is a hallmark of quantum systems, and is in sharp contrast to the diffusive heat transfer mechanisms in classical media where a temperature wave is damped over a distance of the order of its own wavelength [Landau \& Lifshitz, 1987].

\subsubsection{Hamiltonian representation of hydrodynamic equations for the superfluid liquid in a cavity}

As we have seen above, the dynamics of a superfluid liquid can be described, to a first approximation, as the propagation of the density and entropy waves. In what follows, we disregard modes that are localized at the fluid boundaries. (For a detailed consideration of nonlinear phenomena at the surface of quantum liquids, see [Khalatnikov et al., 1995; Kolmakov et al., 2009; Abdurakhimov et al., 2009]). At finite wave amplitude, however, nonlinear effects in the wave interaction play an important role. In particular, second sound is characterized by rather strong nonlinear properties [Dessler \& Fairbank, 1956; Osborne, 1950]. As mentioned above in Sec. 1, the velocity of a traveling second sound wave depends on its amplitude, in accord with Eq. (2).

To describe nonlinear effects, we now introduce the Hamiltonian variables for the system (6). An arbitrary flow of superfluid can be described by three pairs of conjugate variables $(\alpha, \rho),(\beta, S)$ and $(\gamma, f)$ [Pokrovskii \& Khalatnikov, 1976; Khalatnikov et al., 1995]. Here $\alpha$ is the superfluid velocity potential, $\beta$ is the phase variable conjugated to $S$, and $\gamma$ and $f$ are Clebsch variables. The latter define the vorticity in the system; we focus below on potential motion of the superfluid, so we set $\gamma=f=0$. The Hamiltonian function of the superfluid system is given by the total energy expressed in the stationary frame of reference,

$$
H=\int d^{3} \boldsymbol{r}\left[\frac{\rho_{n}}{2} \boldsymbol{v}_{n}^{2}+\frac{\rho_{s}}{2} \boldsymbol{v}_{s}^{2}+\varepsilon(\rho, S, \boldsymbol{p})\right] .
$$

Here $\varepsilon(\rho, S, \boldsymbol{p})$ is the energy per unit volume of the superfluid component in the reference frame moving with the velocity $\boldsymbol{v}_{s}$, and $\boldsymbol{p}$ is the momentum of relative motion of the normal component. The total mass flux is expressed through these variables as $\mathbf{j}=\rho \mathbf{v}_{s}+\boldsymbol{p}=\rho \nabla \alpha+S \nabla \beta$. The equations of superfluid hydrodynamics (6) for the Hamiltonian function (8) are derived in terms of the conjugate Hamiltonian variables as follows [Pokrovskii \& Khalatnikov, 1976; Khalatnikov et al., 1995]

$$
\dot{\rho}=\frac{\delta H}{\delta \alpha}, \quad \dot{\alpha}=-\frac{\delta H}{\delta \rho}, \quad \dot{S}=\frac{\delta H}{\delta \beta}, \quad \dot{\beta}=-\frac{\delta H}{\delta S} .
$$


In Eqs. (9), $\delta$ denotes the variational derivative [Ramon, 1981]. In this representation, the momentum of the relative motion of the normal component is equal to $\boldsymbol{p}=S \nabla \beta$.

If the sound waves propagate in an unrestricted superfluid system, the conjugate variables can be expressed in terms of normal coordinates - the normalized amplitudes of the running first and second sound waves [Pokrovskii \& Khalatnikov, 1976]. In a cavity, the normal modes satisfy the corresponding boundary conditions. In the important case of a high-quality resonator [Borisenko et al., 1988; Kolmakov et al., 2006; Ganshin et al., 2008a; Efimov et al., 2008b; Ganshin et al., 2010], the normal modes are standing waves of first and second sound whose frequencies are equal to the resonant frequencies, $\Omega_{k}=u_{1}\left|\boldsymbol{k}_{n}\right|, \omega_{k}=u_{2}\left|\boldsymbol{k}_{n}\right|$, where $\boldsymbol{k}_{n}$ is the $n$th resonant wave vector.

Variations of the Hamiltonian variables in a wave relative to their equilibrium values are expressed via the sound amplitudes as [Pokrovskii \& Khalatnikov, 1976; Khalatnikov et al., 1995; Brazhnikov et al., 2004]

$$
\begin{array}{ll}
\alpha(\boldsymbol{r}, t)=\sum_{\boldsymbol{k}} \bar{\alpha} \varphi_{\boldsymbol{k}}(\boldsymbol{r})\left(a_{\boldsymbol{k}}-a_{\boldsymbol{k}}^{*}\right), & \delta \rho(\boldsymbol{r}, t)=\sum_{\boldsymbol{k}} \bar{\rho} \varphi_{\boldsymbol{k}}(\boldsymbol{r})\left(a_{\boldsymbol{k}}+a_{\boldsymbol{k}}^{*}\right), \\
\beta(\boldsymbol{r}, t)=\sum_{\boldsymbol{k}} \bar{\beta} \varphi_{\boldsymbol{k}}(\boldsymbol{r})\left(b_{\boldsymbol{k}}-b_{\boldsymbol{k}}^{*}\right), & \delta S(\boldsymbol{r}, t)=\sum_{\boldsymbol{k}} \bar{S} \varphi_{\boldsymbol{k}}(\boldsymbol{r})\left(b_{\boldsymbol{k}}+b_{\boldsymbol{k}}^{*}\right),
\end{array}
$$

where the normal coordinates $a_{\boldsymbol{k}}$ and $b_{\boldsymbol{k}}$ are the complex amplitudes of the first and second sound waves, respectively, $\bar{\rho}, \bar{S}, \bar{\alpha}$ and $\bar{\beta}$ are the normalizing factors, and a star stands for the complex conjugate. The complex amplitudes are used to take account of the phase of the wave. The spatial basis functions depend on the geometry of the cavity and the boundary conditions. For the simplest case of a high quality rectangular resonator, they are $\varphi_{\boldsymbol{k}}(\boldsymbol{r})=\cos \left(k_{x} x\right) \cos \left(k_{y} y\right) \cos \left(k_{z} z\right)$. The equations of motion (6) are represented by the Hamiltonian equations for the sound amplitudes as follows:

$$
i \dot{a}_{\boldsymbol{k}}=\frac{\partial H}{\partial a_{\boldsymbol{k}}^{*}}-i \gamma_{\boldsymbol{k}}^{(1)} a_{\boldsymbol{k}}+F_{\boldsymbol{k}}^{(1)}, \quad i \dot{b}_{\boldsymbol{k}}=\frac{\partial H}{\partial b_{\boldsymbol{k}}^{*}}-i \gamma_{\boldsymbol{k}}^{(2)} b_{\boldsymbol{k}}+F_{\boldsymbol{k}}^{(2)}
$$

Dissipation $\gamma_{\boldsymbol{k}}^{(i)}$, and interaction with an external driving force $F_{\boldsymbol{k}}^{(i)}$, are included phenomenologically [Pokrovskii \& Khalatnikov, 1976; Khalatnikov et al., 1995]; the superscript $i=1,2$ labels the first and second sound modes, respectively. Note that this representation provides a quasiclassical limit for the equations of motion of a superfluid system that can be used provided that the occupation numbers of the corresponding states are sufficiently large, i.e. $\left|a_{\boldsymbol{k}}\right|^{2},\left|b_{\boldsymbol{k}}\right|^{2} \gg \hbar$. However, for the purely quantum case $\left|a_{\boldsymbol{k}}\right|^{2}$, $\left|b_{\boldsymbol{k}}\right|^{2} \sim \hbar$, the normal variables must be considered as operators in a proper Hilbert space.

The nonlinear dynamics of the system (11) can be considered in terms of an expansion of the Hamiltonian $H$ in a Taylor series over the wave amplitudes $a_{\boldsymbol{k}}$ and $b_{\boldsymbol{k}}, H=H_{2}+H_{3}+\ldots$. The term $H_{2}$ is a quadratic function of the amplitudes, $H_{2}=\sum_{\boldsymbol{k}}\left(\Omega_{\boldsymbol{k}}\left|a_{\boldsymbol{k}}\right|^{2}+\omega_{\boldsymbol{k}}\left|b_{\boldsymbol{k}}\right|^{2}\right)$, which describes the propagation of linear waves [Zakharov et al., 1992]. Nonlinear effects, such as the parametric generation and decay of waves, correspond to anharmonic terms in the Hamiltonian, with the coefficients in the expansion over $a_{\boldsymbol{k}}$ and $b_{\boldsymbol{k}}$ being the amplitudes of the processes. Three-wave processes include $(a)$ the decay of the first sound into two second sound waves, $(b)$ Cerenkov emission of the second sound wave by the first sound wave, and $(c)$ inner decay and confluence of waves that belong to the same wave mode. These processes are responsible for the generation of the direct and inverse energy cascades in frequency space for the superfluid. In actual experimental regimes, a single one of these processes may sometimes dominate [Kolmakov et al., 2006; Ganshin et al., 2008a; Nemirovskii, 1990; Rinberg et al., 1996; Rinberg \& Steinberg, 2001; Davidowitz et al., 1995] as also discussed below in Sec. 4.3. We will disregard interactions which involve four or more waves. Therefore, the higher order terms in the expansion of the Hamiltonian over the wave amplitudes are omitted. These higher-order processes are responsible for the isotropisation in the system [Landau \& Khalatnikov, 1949; Khalatnikov, 1950] and should be taken into account in a more general theory. We also disregard mutual transformations between first and second sound waves. 


\subsection{Instability and bifurcation in the system of nonlinear second sound waves}

The experiments have shown that nonlinearity plays essential role in formation of the direct and inverse cascade. To seek understanding of the peculiarities of these transient processes we have undertaken analytical and numerical studies of the dynamics of the nonlinear wave system. Full details of the techniques have been given elsewhere [Brazhnikov et al., 2004; Kolmakov et al., 2006] but, in essence, it involves direct integration of the nonlinear equations (11), expanded up to quadratic terms in the wave amplitude. This numerical technique is somewhat similar to that used by Dyachenko \& Zakharov [2005] for consideration of nonlinear waves on the fluid surface. The techniques allow one to capture accurately the shape of the wave at large amplitudes and/or gradients [Zakharov, 1968]. In the quadratic approximation, the equations of motion (11) governing energy balance in the system are

$$
i \frac{\partial b_{n}}{\partial t}=\sum_{n_{1}, n_{2}} V_{n, n_{1}, n_{2}}\left(b_{n_{1}} b_{n_{2}} \delta_{n-n_{1}-n_{2}}+2 b_{n_{1}} b_{n_{2}}^{*} \delta_{n_{1}-n_{2}-n}\right)-i \gamma_{n} b_{n}+F,
$$

where: $V_{n, n_{1}, n_{2}} \propto \alpha\left(n n_{1} n_{2}\right)^{1 / 2}$ describes the three-wave interaction of the second sound waves; $\gamma_{n}=\nu n^{2}$ models the viscous damping of second sound; and $F \propto W$ is the amplitude of the force driving the $n$-th resonant mode. In this representation the wave spectrum can be calculated as $A_{f} \propto B_{n}\left(b_{n}+b_{n}^{*}\right)$, as follows, starting from Eqs. (10).

Before we present the results of full-scale simulations (Sec.4.3), we consider a reduced model where the mutual interaction of two waves only due to nonlinearity is taken into account. Through this simplified consideration, we demonstrate the key role played by the wave system's instabilities in the formation of high-frequency and low-frequency cascades.

\subsubsection{Instability of nonlinear waves and generation of high frequency harmonics}

It is known that the problem of nonlinear wave propagation and interaction can be mapped onto the problem of coupled oscillator dynamics (Zakharov et al. [1992] describe the general formalism). Based on quite general considerations [L'vov \& Cherepanov, 1982], we may expect the production of waves in the low-frequency domain to be initiated at a frequency close to half that at which the energy is injected into the system. Accordingly, to establish the conditions for the wave instability, we consider a simplified problem where just two interacting wave modes are taken into account. In this case the model Hamiltonian is

$$
H=\omega_{1}\left|b_{1}\right|^{2}+\omega_{2}\left|b_{2}\right|^{2}+\lambda b_{1}^{2} b_{2}^{*}+\lambda b_{1}^{* 2} b_{2} .
$$

Here $\omega_{1}$ and $\omega_{2}$ are the resonant frequencies of second sound waves of infinitely small amplitude, $b_{1}$ and $b_{2}$ are normal coordinates (the amplitudes of the second sound waves), and $\lambda$ is the amplitude of three-wave interaction, proportional to the nonlinearity coefficient of second sound $\alpha_{2}$. The two terms cubic in the wave amplitude describe the interaction of waves due to nonlinearity [Kolmakov, 2003]. The subscript "1" labels the wave of lower frequency (the low-frequency, or LF wave), and subscript "2" labels the wave of higher frequency (the HF wave), i.e. $\omega_{1}<\omega_{2}$. To simplify the analysis, we suppose that $\omega_{1}=\omega_{2} / 2$. We also assume that an external driving force is applied to the system.

The equations of motion (11) for the amplitudes of weakly dissipative second sound waves with the Hamiltonian (13) are

$$
i \dot{b}_{1}=\frac{\partial H}{\partial b_{1}^{*}}-i \gamma_{1} b_{1}+F_{1}(t), \quad i \dot{b}_{2}=\frac{\partial H}{\partial b_{2}^{*}}-i \gamma_{2} b_{2}+F_{2}(t),
$$

where $\gamma_{1}$ and $\gamma_{2}$ are the damping coefficients of second sound waves, and $F_{1}(t)$ and $F_{2}(t)$ are the components of the driving force applied at a frequency $\omega_{1}$,

$$
F_{1}(t)=F_{1} \exp \left(-i \omega_{d} t\right), \quad F_{2}(t)=F_{2} \exp \left(-i \omega_{d} t\right),
$$

$F_{1}$ and $F_{2}$ are the time-independent driving amplitudes, and the dot denotes a derivative with respect to time $t$. 
For further analysis it is convenient to use an interaction representation for the wave amplitudes,

$$
b_{1}=A_{1} \exp \left(-i \omega_{d} t / 2\right), \quad b_{2}=A_{2} \exp \left(-i \omega_{d} t\right)
$$

where $A_{1}$ and $A_{2}$ are new variables. In steady state, $A_{1}$ and $A_{2}$ are time-independent. In this case the equations (14) read

$$
\begin{gathered}
0=i \dot{A}_{1}=-\frac{1}{2}\left(\Delta \omega+2 i \gamma_{1}\right) A_{1}+2 \lambda A_{1}^{*} A_{2}+F_{1}, \\
0=i \dot{A}_{2}=-\left(\Delta \omega+i \gamma_{2}\right) A_{2}+\lambda A_{1}^{2}+F_{2} .
\end{gathered}
$$

Here $\Delta \omega=\omega_{d}-\omega_{2}$ is the detuning of the driving force with respect to the resonant frequency of the HF wave.

We consider, first, the situation where the driving force is applied at a frequency close to the resonant frequency $\omega_{1}$ (low-frequency driving). In the case of a high-quality resonator, $Q \gg 1$, we can omit in Eq. (17) the component $F_{2}$ of the driving force, that acts on the high-frequency mode. In this case, the amplitude $A_{2}$ can be eliminated from Eqs. (16), (17). As a result, one has the following solution for the system:

$$
A_{1}=q F_{1}, \quad q^{-1}=\frac{1}{2}\left(\Delta \omega+2 i \gamma_{1}\right)-\frac{2 \lambda^{2}\left|A_{1}\right|^{2}}{\left(\Delta \omega+i \gamma_{2}\right)}
$$

In the limit of small wave amplitudes, $\left|A_{1}\right| \ll\left|\left(\Delta \omega+2 i \gamma_{1}\right)\left(\Delta \omega+i \gamma_{2}\right)\right| / \lambda$, the equation (18) tends to the well-known relationship between the driving force and amplitude of a linear oscillator [Landau \& Lifshitz, 1976]. For nonlinear waves, the amplitude $A_{2}$ of a high frequency harmonic can be found from (17),

$$
A_{2}=\frac{\lambda A_{1}^{2}}{\Delta \omega+i \gamma_{2}}
$$

It can be seen from Eqs. (18) and (19) that, at an arbitrarily small amplitude of the low-frequency driving force $F_{1}$, the steady-state amplitude of a high-frequency harmonic $A_{2}$ is non-zero. This is a manifestation of the known fact ([Zakharov et al., 1992]) that a nonlinear wave is unstable against generation of high-frequency harmonics due to its nonlinearity. Equations (18), (19) generalize this conclusion to the case of waves with finite viscous damping. As we see, the instability results in the excitation of a wave of frequency twice that of the initial wave, that is $\omega \rightarrow 2 \omega$. The development of the high-frequency wave spectrum (Fig. 2 in Sec. 3.1) with increasing driving force amplitude results from the cascade-like development of this instability, $\omega \rightarrow 2 \omega \rightarrow 3 \omega \ldots$. It is worthy of note that, at high wave amplitudes, the interaction between all waves obeying the resonant condition (3) become important. This explains the formation of wave harmonics at frequencies $n \times \omega$ with odd numbers $n$.

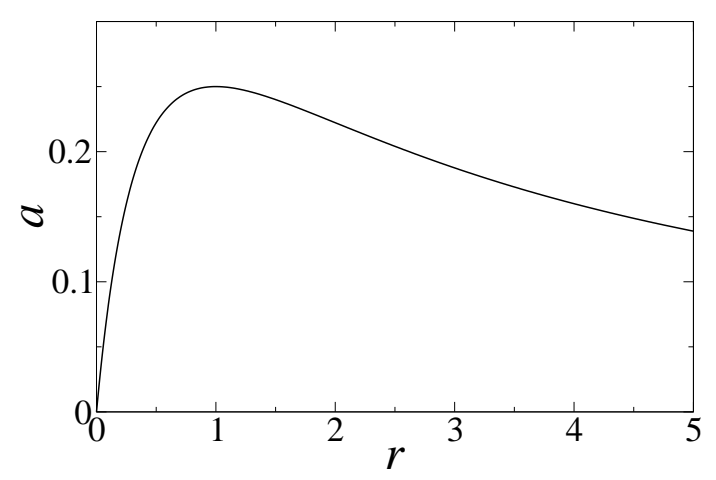

Fig. 8. Dependence of the $a$ parameter on the ratio $r=Q_{1} / Q_{2}$ of the quality factors at the frequencies of the LH and HF second sound waves. 


\subsubsection{Generation of low frequency waves near the instability threshold}

In this section, we consider the process opposite to that described in Sec. 4.2 .1 above, namely, the formation of subharmonics that is, the waves with frequencies lower that the driving frequency. For that, we suppose that the driving frequency $\omega$ is close to the frequency $\omega_{2}$ in Eqs. (18) - (19). In this case, we can omit $F_{1}$ in Eq. (18) at $Q \gg 1$.

Similarly to the consideration given above, the amplitude of the HF wave, $A_{2}$, can be eliminated from the resultant set of equations (16), (17). The equation for the amplitude of the LF wave in steady-state then reads

$$
\left(\Delta \omega^{2}-2 \gamma_{1} \gamma_{2}-4 \lambda^{2}\left|A_{1}\right|^{2}\right)^{2}+\Delta \omega^{2}\left(\gamma_{1}+2 \gamma_{2}\right)^{2}=16 \lambda^{2}|F|^{2}
$$

For convenience we introduce dimensionless variables for the wave amplitude, driving force, detuning, and damping:

$$
\xi_{1}=\frac{2 \lambda\left|A_{1}\right|}{\gamma_{2}+2 \gamma_{1}}, \quad f=\frac{4 \lambda|F|}{\left(\gamma_{2}+2 \gamma_{1}\right)^{2}}, \quad \Delta=\frac{\omega-\omega_{2}}{\gamma_{2}+2 \gamma_{1}}, \quad a=\frac{2 \gamma_{1} \gamma_{2}}{\left(\gamma_{2}+2 \gamma_{1}\right)^{2}} .
$$

In terms of these dimensionless variables the equation (20) becomes

$$
\xi_{1}=\left[\left(f^{2}-\Delta^{2}\right)^{1 / 2}+\Delta^{2}-a\right]^{1 / 2}
$$

The parameter $a$ is expressed in terms of the quality factor of the resonator $Q_{1}=\omega_{1} / \gamma_{1}$ and $Q_{2}=\omega_{2} / \gamma_{2}$ at frequencies equal to the frequencies of LF and HF waves as follows

$$
a=\frac{Q_{1} Q_{2}}{\left(Q_{1}+Q_{2}\right)^{2}}
$$

Dependence of the $a$ parameter on the ratio $r=Q_{1} / Q_{2}$ is shown in Fig. 8 .

The dependence $a(r)$ has a maximum $a=1 / 4$ at $r=1$. Assuming that the frequency dependence of the damping coefficient for second sound takes the form $\gamma(\omega)=\nu_{0} \omega^{2}$, which is typical of sound waves in an unrestricted liquid, we may evaluate the $a$ coefficient as (recall here that $\omega_{1} \approx \omega_{2} / 2$ )

$$
\gamma_{1} / \gamma_{2}=0.25, \quad a \approx 0.2
$$

that is, close to its maximum value. It follows from Eq. (22) that the amplitude of the LH wave is nonzero only if the amplitude of the driving force at the frequency of HF wave exceeds the critical value $f_{c}=$ $\max \left(f_{c 1}, f_{c 2}\right)$, and if $f_{c 1}$ and $f_{c 2}$ obey the following conditions:

$$
\begin{gathered}
f_{c 1}-|\Delta|=0 \\
\left(f_{c 2}^{2}-\Delta^{2}\right)^{1 / 2}+\Delta^{2}-a=0 .
\end{gathered}
$$

The dependence of the critical amplitude of the driving force $f_{c}$ on the detuning $\Delta$, defined by the formulas (23) and (24), is shown in Fig. 9(left).

It follows from subsequent analysis that, in the area inside the rectangle $\left(\Delta \mid<a^{1 / 2}, a<f<a^{1 / 2}\right)$ in Fig. 9(left), the generation of the LH wave is analogous to a second order phase transition, because the amplitude $\xi_{1}$ starts from zero. At $f>a^{1 / 2}$ the generation transition of the LH frequency is of the hard type, however, analogous to a first order transition, and it is accompanied by a finite jump in wave amplitude.

To demonstrate this, we consider the dependence of the amplitude of the LF wave $\xi_{1}$ on the detuning $\Delta$ for a given value of the driving force $f$. At $f \leq f_{c \min }=a$ the amplitude $\xi_{1}$ is equal to zero. At $f>f_{c \min }$ there are two possibilities: in accordance with Eq. (22) the second derivative the amplitude $\xi_{1}$ with respect to $\Delta$ taken at $\Delta=0$ is equal to

$$
\left.\frac{d^{2} \xi_{1}}{d \Delta^{2}}\right|_{\Delta=0}=\frac{1-2 f}{2 f(f-a)^{1 / 2}}
$$



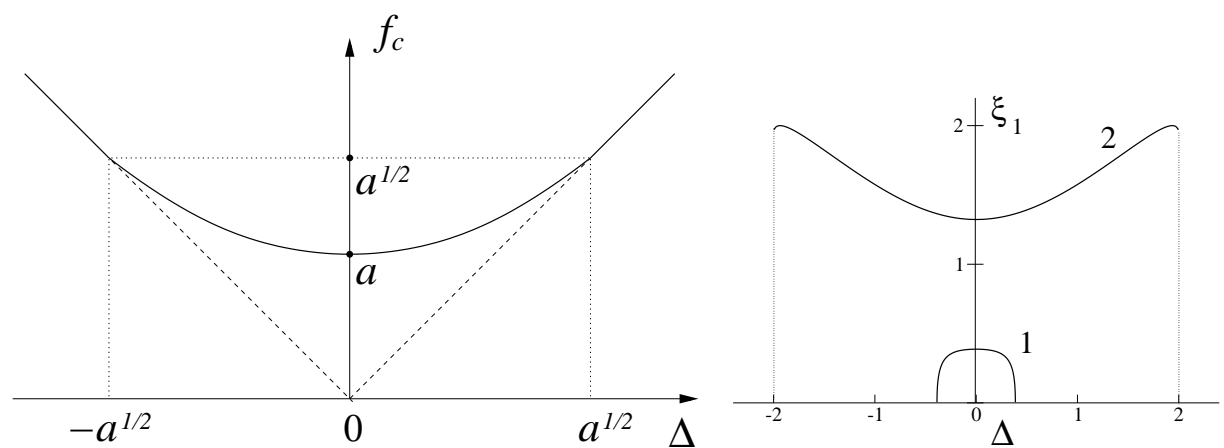

Fig. 9. Left: calculated dependence of the critical driving force amplitude on the detuning $\Delta$ from the second sound resonant frequency. Right: amplitude of the LF wave plotted as a function of detuning for two driving amplitudes, $f=0.4$ (i.e. $f<a^{1 / 2}$, curve 1$)$, and $f=2\left(f>a^{1 / 2}\right.$, curve 2$)$. The damping is $a=1 / 4$.

If $a<f<a^{1 / 2}$, the second derivative (25) is negative, and the amplitude $\xi_{2}$ at half the driving frequency decreases with increasing $\Delta$ (curve 1 in the right plot of Fig. 9). Recall that $a$ is always less than unity. For the case $f>a^{1 / 2}$ the wave amplitude $\xi_{2}$ rises with an increase in the detuning (curve 2).

It follows from this analysis that, at small or zero detuning, the second sound wave system is stable against generation of subharmonics but only when $f \leq a$. At higher driving amplitudes, $f>a$, the solution of equations (18) - (19) at $A_{1}=A_{2}=0$ becomes unstable against small low-frequency perturbation. In this regime, subharmonics are generated. This wave instability is similar to the Hopf bifurcation of coupled oscillators [Zhan et al., 2002].

The formation of a single wave of frequency equal to half the driving frequency is clearly seen in experiments: see Fig. 3(b). Generation of multiple subharmonics for sufficiently high amplitude of the driving force (Fig.5) can be understood as a cascade of decays of non-linearly generated waves towards the low-frequency region. The dependence of the total energy content of the low-frequency subsystem Fig. 6 obtained from the experimental data confirms our conclusion about the threshold character of the instability against generation of subharmonics.

To estimate the critical wave amplitude above which generation of the low-frequency wave becomes possible, we take $\xi_{1} \sim 1$, and [Kolmakov, 2003]

$$
\lambda=\sqrt{\frac{\rho_{s}}{\rho \rho_{n}}} \frac{\alpha \sigma \omega^{3 / 2}}{6 \sqrt{2} u_{2}(\partial \sigma / \partial T)}, \quad \delta T=\sqrt{\frac{\omega}{(\partial S / \partial T)}}|A|, \quad|A|=\frac{\left(\gamma_{2}+2 \gamma_{1}\right)}{2 \lambda} \xi_{1}, \quad u_{2}^{2}=\frac{\rho_{s} \sigma^{2}}{\rho_{n}(\partial \sigma / \partial T)}
$$

From here one can estimate the critical second sound wave amplitude as $\delta T \sim \gamma /|\alpha| \omega=1 /|\alpha| Q$, where $\gamma$ is the damping coefficient of the second sound wave, and $Q$ is the quality factor of the resonator. Taking the values $Q \sim 10^{3},|\alpha| \sim 7.7 K^{-1}$ one has $\delta T \sim 0.1 \times 10^{-3} \mathrm{~K}$ by order of magnitude, which is consistent with the experimental observations.

\subsection{Numerical simulations}

\subsubsection{Second sound turbulence}

To capture the details of the formation processes of the wave cascades, we have undertaken a numerical study of the dynamics of nonlinear waves in superfluid ${ }^{4} \mathrm{He}$ within the high- $Q$ resonator in terms of the full equation (12). We use model parameters corresponding to those in the experiments [Kolmakov et al., 2006; Ganshin et al., 2008a, 2010]. The latter were conducted at temperatures relatively close to the temperature of the superfluid transition, $\left(T_{\lambda}-T\right) / T_{\lambda}<0.05$. As pointed out in Sec. 4.1.2, the nonlinearity coefficient $\alpha_{2}$ grows as $T \rightarrow T_{\lambda}$ and hence the interaction between the second sound waves becomes important. On the other hand, the interaction between second sound and first sound is relatively small in this regime and can to a first approximation be neglected. Accordingly, the simulations only take account of terms containing the second sound amplitudes $b_{\boldsymbol{k}}$.

The frequency of second sound depends on its wave vector $k$ as [Tyson \& Douglass, Jr., 1968] given 

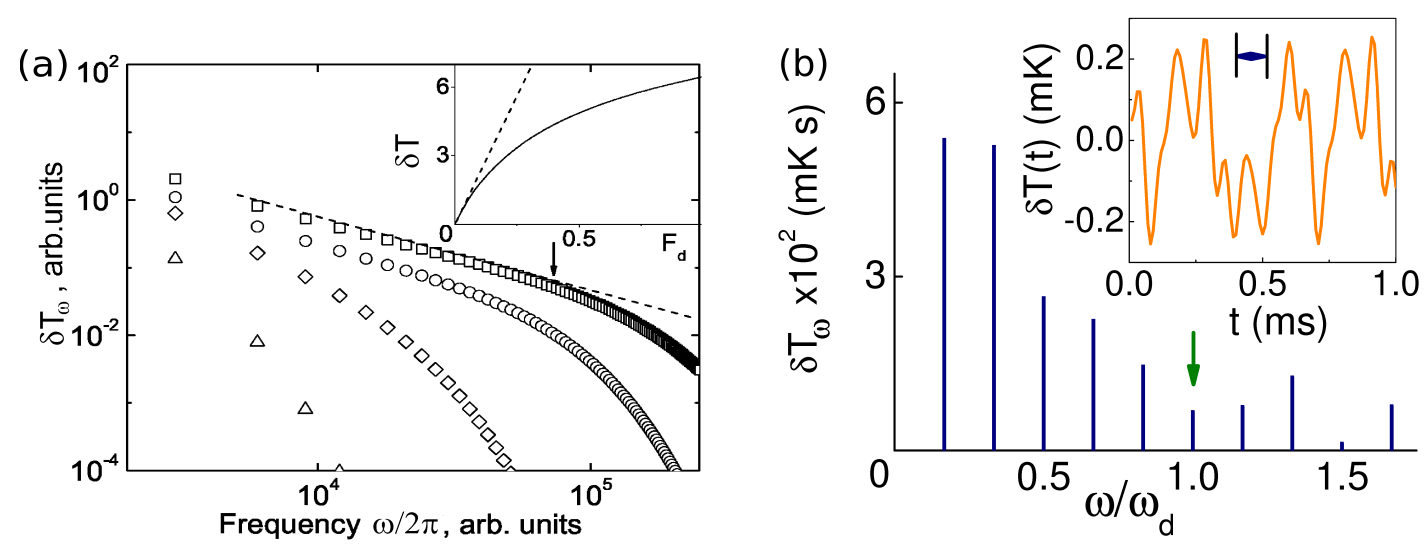

Fig. 10. (a) Steady-state second sound power spectral amplitudes $\delta T_{\omega}$ calculated numerically from Eq. (12) for four different driving force amplitudes: $F_{d}=0.01$ (triangles); 0.05 (diamonds); 0.1 (circles); and 0.3 (squares). The dashed line corresponds to $\delta T_{\omega} \propto \omega^{-1}$. The arrow marks the boundary frequency $\omega_{b} / 2 \pi$ of the inertial range for $F_{d}=0.3$. The inset shows the calculated dependence on $F_{d}$ of the standing wave amplitude $\delta T$ : solid line - nonlinear waves, $\alpha_{2}\left\langle 0\left(T>T_{\alpha}\right)\right.$; dashed line - linear waves, $\alpha_{2}=0\left(T=T_{\alpha}\right)$. (From Kolmakov et al. [2006].) (b) Formation of the inverse cascade in the second sound wave spectrum computed for $t=1000$. Main plot: the wave spectrum in the presence of the inverse cascade computed for $N=10$ modes in the resonator. The vertical arrow indicates the frequency $\omega_{\text {drive }}$ of the driving force. Inset: oscillations of temperature at a wall of the resonator. The horizontal arrow shows the driving-force period.

by Eq. (2). As we mention in Sec. 1, the dispersion of second sound is significant within a close vicinity of the superfluid transition (i.e. for $T_{\lambda}-T<1 \mu \mathrm{K}$ ) but is very weak in the temperature range $T<2.1 \mathrm{~K}$. However, the presence of non-zero dispersion is of key importance for the wave dynamics in the superfluid. It is well established that, if the dispersion is exactly equal to zero, $\partial u_{2} / \partial k=0$, the main mechanism of energy transport through the frequency scales is associated with the creation of a shock wave [Kadomtsev $\&$ Petviashvili, 1970]. When the dispersion is positive but small, $\partial u_{20} / \partial k>0$ (e.g. in the case of second sound in He II), the resonant three-wave interaction is only relevant within a narrow cone of k-vectors around the direction of propagation of the wave with $\mathbf{k}=\mathbf{k}_{\text {drive }}$ [Zakharov et al., 1992; L'vov et al., 2000]. The relative phases of waves with $\mathbf{k}$-vectors almost collinear to $\mathbf{k}_{\text {drive }}$ (i.e. within the cone) are random, and a kinetic equation for waves can be used to describe the cascade-like propagation of energy in the wave system [Zakharov et al., 1992; Zakharov \& Sagdeev, 1970]. However, the interaction of waves with non-collinear wave vectors is controlled by higher nonlinear terms and is relatively small. That is, the wave distribution in the superfluid is nearly one-dimensional, provided that the dispersion given by Eq. (2) is weak. The formation of this wave regime manifests itself in, for example, fluctuations of the wave field at high frequencies and in the establishment of a near-Gaussian probability distribution function for the second sound wave amplitudes, as was observed by Efimov et al. [2008b]. This regime is a close analogue of classical wave turbulence [Zakharov et al., 1992]. We refer to it as the acoustic turbulence of second sound waves or, the second sound turbulence, see Sec.3.

The nonlinear dynamics of second sound involves the interactions of sound waves on different scales. Hence the full equations (11) should be solved numerically. In these simulations, we consider onedimensional longitudinal second sound waves in the long cylindrical resonator [Kolmakov et al., 2006]. We neglect the possible generation of transverse, radial modes. Note, however, that the excitation of transverse modes in a cylindrical resonator can be important for non-planar, three dimensional waves [Zinov'eva, 1957]. The longitudinal resonant wave vector is $k_{n}=\pi n / L$ where $L$ is the length of the resonator and $n$ is the number of the resonant wave mode. In the present model, wave damping was taken into account at all frequencies, a feature that is of key importance [Kolmakov et al., 2006] for a correct description of the formation of a cascade of nonlinear sound waves with increasing amplitude of the driving force.

Fig. 10(a) shows the evolution of the steady-state spectrum with increasing driving force amplitude $F_{d}$, calculated for $\alpha_{2}<0\left(T>T_{\alpha}\right)$. We used a periodic driving force of frequency equal to a resonant frequency of the resonator corresponding to the conditions of the measurements, taken as $\omega_{\text {drive }} / 2 \pi=3000$ 
in dimensionless units. The effective viscosity coefficient $\nu$ was fitted to the measured value of the quality factor $Q \sim 3 \times 10^{3}$ of the resonator, to facilitate comparison of the model results with those from the experiments [Kolmakov et al., 2006]. Points on the plot correspond to the amplitudes of the peaks in the spectrum. At small driving amplitude $F_{d} \sim 0.01$ (triangles), viscous damping evidently prevails at all frequencies and a turbulent cascade is not formed: the amplitude of the second harmonic is an order less that the amplitude of the main harmonic. In this regime the wave shape is close to sinusoidal. At intermediate driving amplitude $F_{d} \sim 0.05$ (diamonds) nonlinearity starts to play a role at frequencies of the order of driving frequency, and a few harmonics are generated. At high driving amplitudes $F_{d} \geq 0.1$ (circles and squares) a well-developed cascade of second sound waves is formed up to frequencies 30 times that of the driving frequency.

\subsubsection{The inverse cascade}

To capture the main characteristic features of the development of the inverse cascade while, at the same time, keeping the system simple enough to be analyzed in detail, we proceed within the framework of equations (12) taking account of the relatively small numbers of waves $N$ : the summation on the r.h.s. of Eq. (12) was made for $n_{1}, n_{2}$ ranging from 1 to $N$. We present below the results obtained for $N=10$. We assume that a periodic driving force is applied at the 6th resonant frequency.

The simulations show that, for sufficiently high driving amplitude $W$, low frequency harmonics at $\omega<\omega_{\text {drive }}$ are formed in the wave spectrum (see Fig. 10 (b)). Consistent with the discussion above, this is a manifestation of the formation of an inverse cascade in the superfluid. The numerical results describe very well the data obtained in the experiments on the inverse cascade of second sound waves in He-II (see Sec. 3.2 below).

It is clearly evident from Fig. 10(b) that the inverse cascade is responsible for the formation of highamplitude, low-frequency, subharmonics (cf. the experimental results of Fig. 6(right)). As shown by Ganshin et al. [2008a] and Efimov et al. [2010], the inverse cascade develops through the formation of isolated lowfrequency waves of higher amplitude than is typical of the waves around them. These higher-amplitude lone waves can be considered as the acoustic analogue of the rogue waves that occasionally appear on the ocean and endanger shipping.

\section{Conclusions}

The experiments described above have revealed direct and inverse wave energy cascades in a turbulent acoustic system. The onset of the inverse cascade involves a threshold in the excitation level being exceeded, seems to occur through a bifurcation, and is strikingly similar to the Feigenbaum period-doubling route to chaos. The result is a substantial increase in wave amplitude together with the formation of high-amplitude, low-frequency, subharmonics. The instability develops through formation of isolated low-frequency waves of higher amplitude than is typical of the waves around them, repesenting an acoustic analogue of oceanic rogue waves. Their origin lies in the decay instability of the periodic wave, i.e. a similar mechanism to that proposed [Onorato et al., 2001; Dyachenko \& Zakharov, 2005] (modulation instability) to account for the creation of oceanic rogue waves [Dean, 1990]. We have also glimpsed a rich manifold of kinetic phenomena waiting to be explored in future experimental and theoretical investigations.

Finally, we emphasise that these studies of wave turbulence are likely to impact on the several other areas of physics mentioned in Sec. 1. Practical applications can readily be envisaged in relation to the extraction of energy from ocean waves, where we have the possibility of using tailored perturbations to alter the dominant wavelength to match the optimal characteristics of the machinery in use. It is also possible that an improved understanding of how "rogue" waves originate may eventually lead to methods for predicting or attenuating them.

\section{Acknowledgements}

We are grateful to V. E. Zakharov, E. A. Kuznetsov, A. A. Levchenko, M. Yu. Brazhnikov and L. V. Abdurakhimov for valuable discussions. The investigations were supported in part by the National Science 
Foundation U.S.A. through Teradgid grant TG-DMR100103, by the Center for Simulation and Modeling of the University of Pittsburgh, and by the Engineering and Physical Sciences Research Council (U.K.).

\section{References}

Abdurakhimov, L. V., Brazhnikov, M. Y. \& Levchenko, A. A. [2009] "Capillary turbulence on the surface of normal and superfluid ${ }^{4} \mathrm{He}, "$ Low Temp. Phys. 35, 95-99.

Abdurakhimov, L. V., Brazhnikov, M. Y., Remizov, I. A. \& Levchenko, A. A. [2010] "Observation of wave energy accumulation in the turbulent spectrum of capillary waves on the He-II surface under harmonic pumping," JETP Letters 91, 271-276.

Ackerman, C. C., Bertman, B., Fairbank, H. A. \& Guyer, R. A. [1966] "2nd sound in solid helium," Phys. Rev. Lett. 16, 789-791.

Anderson, P. W. \& Suhl, H. [1955] "Instability in the motion of ferromagnets at high microwave power levels," Phys. Rev. 100, 1788-1789.

Atkin, R. J. \& Fox, N. [1985] "Spherical thermal shock waves in Helium II," J. Phys. C: Solid St. Phys. 18, 1585-1593.

Bisnovatyi-Kogan, G. S. \& Silich, S. A. [1995] "Shock-wave propagation in the nonuniform interstellar medium," Rev. Mod. Phys. 67, 661-712.

Borisenko, I. Y., Efimov, V. B. \& P.Mezhov-Deglin, L. [1988] "Nonlinear 2nd sound waves in a liquid helium resonator," Fiz. Nizk. Temp. (USSR) 14, 1123-1126.

Brazhnikov, M. Y., Efimov, V. B., Kolmakov, G. V., Levchanko, A. A., Lebedeva, E. V. \& Mezhov-Deglin, L. P. [2004] "Turbulence of second sound waves in superfluid He II," Low Temp. Phys. 30, 441-445.

Davidowitz, H., Lvov, Y. \& Steinberg, V. [1995] "Burgers' equation and the evolution of nonlinear 2nd sound," Physica D 84,635-644.

Davidson, P. A. [2004] Turbulence: An Introduction for Scientists and Engineers (Oxford University Press, Oxford).

Dean, R. G. [1990] "Freak waves: a possible explanation," Water Wave Kinetics, eds. Torum, A. \& Gudmestad, O. T. (Kluwer, Amsterdam), pp. 609-612.

Dessler, A. J. \& Fairbank, W. H. [1956] "Amplitude dependence of the velocity of second sound," Phys. Rev. 104, 6-12.

Devaney, R. L. [2000] An Introduction to Chaotic Dynamical Systems (Addison-Wesley, Reading).

Dyachenko, A. I. \& Zakharov, V. E. [2005] "Modulation instability of Stokes wave $\rightarrow$ freak wave," JETP Lett. 81, 255-259.

Efimov, V. B., Ganshin, A. N., Kolmakov, G. V., McClintock, P. V. E. \& Mezhov-Deglin, L. P. [2008a] "Wave turbulence in superfluid ${ }^{4} \mathrm{He}$ : Energy cascades and rogue waves in the laboratory," Let's Face Chaos Through Nonlinear Dynamics, Proc. of 7th Intern. Summer School and Conf. in Maribor, eds. Robnik, M. \& Romanovski, V. (AIP Conf. Proc., Melville, NY), pp. 53-62.

Efimov, V. B., Ganshin, A. N., Kolmakov, G. V., McClintock, P. V. E. \& Mezhov-Deglin, L. P. [2009] "Acoustic turbulence in superfluid ${ }^{4}$ He," J. Low Temp. Phys. 156, 95-115.

Efimov, V. B., Ganshin, A. N., Kolmakov, G. V., McClintock, P. V. E. \& Mezhov-Deglin, L. P. [2010] "Rogue waves in superfluid helium," Eur. Phys. J. Special Topics 185, 181-193.

Efimov, V. B., Ganshin, A. N. \& McClintock, P. V. E. [2008b] "Statistical properties of strongly nonlinear waves within a resonator," Phys. Rev. E 78, 066611.

Efimov, V. B., Ganshin, A. N., McClintock, P. V. E., Kolmakov, G. V. \& Mezhov-Deglin, L. P. [2006] "Experimental study of the nonlinear second sound wave interaction in superfluid ${ }^{4} \mathrm{He}, "$ J. Low Temp. Phys. 145, 155-164.

Efimov, V. B., Kolmakov, G. V., Kuliev, A. S. \& Mezhov-Deglin, L. P. [1998] "Propagation of short nonlinear second sound pulses through He-II in one- and three-dimentional geometry," Low Temp Phys. 24, 81-85.

Efimov, V. B., Kolmakov, G. V., Lebedeva, E. V., Mezhov-Deglin, L. P. \& Trusov, A. B. [2000] "Generation of second and first sound waves by a pulse heater in He-II under pressure," J. Low Temperature Phys. 119, 309-322. 
Falkovich, G. \& Meyer, M. [1996] "Two-dimensional acoustic turbulence," Phys. Rev. E 54, 4431-4434.

Frisch, U. [1995] Turbulence (Cambridge University Press, Cambridge).

Ganshin, A. N., Efimov, V. B., Kolmakov, G. V., Mezhov-Deglin, L. P. \& McClintock, P. V. E. [2008a] "Observation of an inverse energy cascade in developed acoustic turbulence in superfluid helium," Phys. Rev. Lett. 101, 065303.

Ganshin, A. N., Efimov, V. B., Kolmakov, G. V., Mezhov-Deglin, L. P. \& McClintock, P. V. E. [2010] "Experiments on wave turbulence: the evolution and growth of second sound acoustic turbulence in superuid ${ }^{4}$ He confirm self-similarity," New J. Phys. 12, 083047.

Ganshin, A. N., McClintock, P. V. E., Efimov, V. B., Kolmakov, G. V. \& Mezhov-Deglin, L. P. [2008b] "Observation of acoustic turbulence in a system of nonlinear second sound waves in superfluid He-4," Low Temp. Phys. 34, 288-292.

Goldner, L. S., Ahlers, G. \& Mehrotra, R. [1991] "Quantitative studies of nonlinear 2nd sound in superfluid ${ }^{4}$ He," Phys. Rev. B 43, 12861.

Gurbatov, S. N., Kurin, V. V., Kustov, L. M. \& Pronchatov-Rubtsov, N. V. [2005] "Physical modeling of nonlinear sound wave propagation in oceanic waveguides of variable depth," Acoust. Phys. 51, $152-159$.

Incropera, F. P. \& DeWitt, D. P. [1981] Fundamentals of Heat Transfer (Wiley, New York).

Iznankin, A. Y. \& Mezhov-Deglin, L. P. [1983] "Shock waves in liquid helium," Sov. Phys. JETP 57, 801-808.

Kadomtsev, B. B. \& Petviashvili, V. I. [1970] "On the stability of solitary waves in weakly dispersive media," Sov. Phys. Doklady 15, 539-541.

Kartashova, E. [2010] Nonlinear Resonance Analysis (Cambridge University Press, Cambridge).

Khalatnikov, I. [1950] "Absorption of sound in helium II," Zh. Eksp. Teor. Fiz. 20, 247-266.

Khalatnikov, I. M. [1965] An Introduction to the Theory of Superfluidity (Benjamin, New York).

Khalatnikov, I. M., Kolmakov, G. V. \& Pokrovsky, V. L. [1995] "Transformation of second sound into surface waves in liquid helium," JETP 80, 873.

Kolmakov, G. V. [2003] "Nonlinear second-sound waves in ${ }^{4} \mathrm{He}-{ }^{3} \mathrm{He}$ superfluid mixtures," Low Temp. Phys. 29, 495-498.

Kolmakov, G. V., Brazhnikov, M. Y., Levchenko, A. A., Abdurakhimov, A. V., McClintock, P. V. E. \& Mezhov-Deglin, L. P. [2009] "Capillary turbulence on the surface of quantum fluids," Progress in Low Temperature Physics: Quantum Turbulence, eds. Halperin, W. P. \& Tsubota, M. (Elsevier, Amsterdam), pp. 303-349.

Kolmakov, G. V., Efimov, V. B., Ganshin, A. N., McClintock, P. V. E. \& Mezhov-Deglin, L. P. [2006] "Formation of a direct Kolmogorov-like cascade of second sound waves in He II," Phys. Rev. Lett. 97, 155301.

Kolmakov, G. V., Mezhov-Deglin, L., Efimov, V. \& Lebedeva, E. [2004] "Nonlinearity and interaction of second sound waves in superfluid ${ }^{4} \mathrm{He}-{ }^{3} \mathrm{He}$ mixture," Physica Status Solidi C 1, 3007-3010.

Kolmogorov, A. N. [1941] "Local structure of turbulence in incompresible liquid at very large Reynolds numbers," Doklady Akad. Nauk S.S.S.R. 30, 299.

Landau, L. \& Khalatnikov, I. [1949] "Theory of viscosity of helium II. I. Collisions of elementary excitations in helium II," Zh. Eksp. Teor. Fiz. 19, 637-650.

Landau, L. D. \& Lifshitz, E. M. [1976] Mechanics (Pergamon, New York).

Landau, L. D. \& Lifshitz, E. M. [1987] Fluid Mechanics (Butterworth and Heinemann, Oxford).

Lifshitz, E. M. [1944] "Radiation of sound in helium II," Sov. Phys. JETP 14, 116.

L'vov, V., L'vov, Y. \& Pomyalov, A. [2000] "Anisotropic spectra of acoustic turbulence," Phys. Rev. E 61, $2586-2594$.

L'vov, V. S. [1994] Wave Turbulence under Parametric Excitation. Applications to Magnets (Springer, Berlin).

L'vov, V. S. \& Cherepanov, V. B. [1982] "Nonlinear theory of the "kinetic" excitations of waves," Sov. Phys. JETP 54, 746-754.

Matsushita, T., Nomura, R., Hensley, H. H., Shiga, H. \& Mizusaki, T. [1996] "Spin dynamics and onset of Suhl instability in bcc solid ${ }^{3} \mathrm{He}$ in the nuclear-ordered U2D2 phase," J. Low Temp. Phys. 105, 
67-92.

McCintock, P. V. E., Efimov, V. B., Ganshin, A. N., Kolmakov, G. V. \& Mezhov-Deglin, L. P. [2008] "Turbulence of second sound waves in superfluid ${ }^{4} \mathrm{He}$ effect of low frequency resonant perturbations," J. Low Temp. Phys. 150, 394-401.

Mezhov-Deglin, L. P., Iznankin, A. Y. \& Mineev, V. P. [1980] "Observation of 2nd sound rarefaction shock waves in superfluid helium," JETP Lett. 32, 199-203.

Nemirovskii, S. K. [1990] "Nonlinear acoustics of superfluid helium," Soviet Phys. Usp. 33, 429-452.

Onorato, M., Osborne, A. R., Serio, M. \& Bertone, S. [2001] "Freak waves in random oceanic sea states," Phys. Rev. Lett. 86, 5831-5834.

Osborne, D. V. [1950] "The rotation of liquid helium II," Proc. Phys. Soc. 63, 909-912.

Osborne, D. V. [1951] "2nd sound in liquid helium II," Proc. Phys. Soc. (Lond.) A 64, 114-123.

Pokrovskii, V. L. \& Khalatnikov, I. M. [1976] "Transformation of first sound into second in superfluid helium," Sov. Phys. JETP 44, 1036.

Pushkarev, A. N. \& Zakharov, V. E. [1996] "Turbulence of capillary waves," Phys. Rev. Lett. 76, 3320-3323.

Putterman, S. J. [1974] Superfluid Hydrodynamics (North-Holland, Amsterdam).

Ramon, P. [1981] Field Theory: a Modern Primer (Benjamin-Cunnings, Reading, MA).

Rinberg, D., Cherepanov, V. \& Steinberg, V. [1996] "Parametric generation of second sound by first sound in superfluid helium," Phys. Rev. Lett. 76, 2105-2108.

Rinberg, D. \& Steinberg, V. [2001] "Parametric generation of second sound by first sound in superfluid helium," Phys. Rev. B 64, 054506.

Ryutova, M. \& Tarbell, T. [2003] "MHD shocks and the origin of the solar transition region," Phys. Rev. Lett. 90, 191101.

Solli, D. R., Ropers, C., Koonath, P. \& Jalali, B. [2007] "Optical rogue waves," Nature 450, 1054-1057.

Spangler, S. R., Leckband, J. A. \& Cairns, I. H. [1997] "Observations of the parametric decay instability of nonlinear magnetohydrodynamic waves," Phys. Plasmas 4, 846-855.

Temperley, H. N. V. [1951] "The theory of the propagation in liquid helium-II of temperature waves of finite amplitude," Proc. Phys. Soc. (Lond.) A 64, 105-114.

Tsoi, V. S. [2003] "Transverse electron focusing as a way of studying phonon kinetics. Turbulence of phonon flow," CEJP 1, 72-90.

Tyson, J. A. \& Douglass, Jr., D. H. [1968] "Critical-region second-sound velocity in He II," Phys. Rev. Lett. 21, 1308-1310.

Vinen, W. F. [2006] "An introduction to quantum turbulence," J. Low Temp. Phys. 145, 7-24.

Vinen, W. F. \& Niemela, J. J. [2002] "Quantum turbulence," J. Low Temp. Phys. 128, 167-231.

Zakharov, V. E. [1968] "Stability of periodic waves of finite amplitude on the surface of a deep fluid," $J$. Appl. Mech. Tech. Phys. 9, 190-194.

Zakharov, V. E., L'vov, V. S. \& Falkovich, G. [1992] Kolmogorov Spectra of Turbulence I (Springer, Berlin).

Zakharov, V. E. \& Sagdeev, R. Z. [1970] "On spectrum of acoustic turbulence," Doklady Akad. Nauk S.S.S.R. 192, 297-300.

Zhan, M., Wei, G. W., Lai, C. H., Lai, Y. C. \& Liu, Z. H. [2002] "Coherence resonance near the Hopf bifurcation in coupled chaotic oscillators," Phys. Rev. E 66, 036201.

Zinov'eva, K. N. [1957] "Coefficient of bulk absorption of the second sound and viscosity of the normal component of helium ii down to $0.83 \mathrm{~K}, "$ Sov. Phys. JETP 4, 36-40. 